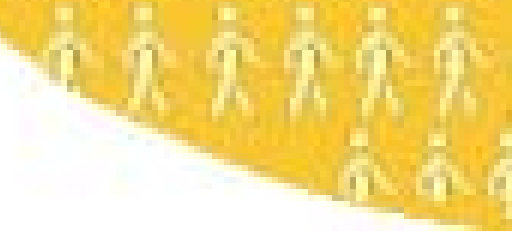

\title{
NONPARAMETRIC IDENTIFICATION UNDER DISCRETE VARIATION
}

Andrew Chesher

THE INSTITUTE FOR FISCAL STUDIES DEPARTMENT OF ECONOMICS, UCL cemmap working paper CWP19/03 


\title{
Nonparametric identification under discrete variation
}

\author{
Andrew Chesher* \\ Centre for Microdata Methods and Practice \\ Institute for Fiscal Studies and University College London
}

December 14th 2003

\begin{abstract}
This paper provides weak conditions under which there is nonparametric interval identification of local features of a structural function which depends on a discrete endogenous variable and is nonseparable in a latent variate. The function may deliver values of a discrete or continuous outcome and instruments may be discrete valued. Application of the analog principle leads to quantile regression based interval estimators of values and partial differences of structural functions. The results are used to investigate the nonparametric identifying power of the quarter of birth instruments used by Angrist and Krueger (1991) in their study of the returns to schooling.
\end{abstract}

\section{INTRODUCTION}

1.1. Discrete variation. This paper explores the limits to identification of structural features when there is discrete variation. Many of the econometric models involving discrete variables used in practice embody parametric restrictions. As persuasively argued by Roehrig (1988), it is important to know the extent to which parametric restrictions make an essential contribution to a model's identifying power. This paper sheds light on this issue by studying the identifying power of nonparametric econometric models when there is discrete variation.

When there is discrete variation parametric restrictions are powerful. That is a matter for concern because parametric restrictions never flow from economic theory. Discrete variation in covariates places limits on the values of endogenous variables at which features of structural functions can be nonparametrically identified and prohibits nonparametric identification of derivatives of structural functions. ${ }^{1}$ Parametric restrictions allow "interpolation" between points at which nonparametric identification is feasible.

\footnotetext{
*I am grateful to Roger Koenker, Charles Manski, and Joao Santos Silva and to seminar participants at cemmap, Northwestern University, University of Chicago and at the Harvard-MIT econometrics workshop for comments on the related paper Chesher (2002) and to Hidehiko Ichimura, Whitney Newey and Elie Tamer for comments on this paper. The paper was presented at the 14th EC $^{2}$ Meeting held at cemmap, London, December 12th - 13th 2003.

${ }^{1}$ See Chesher (2002).
} 
Discrete variation in endogenous variables poses particular problems. It is shown here that point nonparametric identification of values of structural functions is infeasible - at best there is interval identification - unless special circumstances prevail. Consequently, there is generally at best interval nonparametric identification of the effects on structural functions of varying the values of discrete endogenous variables.

The paper provides conditions under which there is interval nonparametric identification of:

1. values of structural functions at interior points of support of a discrete endogenous variable, and,

2. partial differences of structural functions for variation across interior points of support of a discrete endogenous variable.

The exclusion of extreme points of support means that the binary endogenous variable case lies outside the scope of application of the results given here. The demonstration of the identifying power of the proposed model is constructive and points to easily computed analog interval estimators.

1.2. Nonseparable structural functions. A stylised Becker-Chiswick-Mincer model $^{2}$ of the determination of investment in schooling and the wage provides one motivation for studying the problem considered in this paper. Let $Y_{1}, Y_{2}$ and $X$ denote respectively the log wage, years of schooling and a list of covariates and let $\rho_{1}$ and $\rho_{2}$ denote continuously jointly distributed scalar random variables. The structural equations:

$$
\begin{aligned}
& Y_{1}=h_{1}\left(Y_{2}, X, \rho_{1}\right) \\
& Y_{2}=h_{2}\left(X, \rho_{2}\right)
\end{aligned}
$$

deliver a single value of $Y_{1}$ and $Y_{2}$ given a value of $X, \rho_{1}$ and $\rho_{2}$. In this context there is interest in the sensitivity of the structural function $h_{1}$ to variation in $Y_{2}$ when other arguments are held fixed, because this bears on the "returns to schooling". If $\rho_{1}$ and $\rho_{2}$ are correlated this is not identified without further restrictions.

The structural functions (1) and (2) are required to be monotonically varying (normalised increasing), but not additively separable in the latent variates, so the returns to schooling may exhibit random variation driven by the variation in $\rho_{1}$ which may co-vary with $\rho_{2}$. In this situation one might be content to identify some average value of the returns to schooling, but of much greater interest is identification of the value of the returns to schooling at particular values of $Y_{2}, X$ and $\rho_{1}$. This paper focusses on the case in which there is discrete variation in schooling as often arises in practice.

\footnotetext{
${ }^{2}$ See Becker and Chiswick (1966), Chiswick (1974), Chiswick and Mincer (1972) and Mincer (1974). Card (2001) considers parametric nonseparable models of wage determination and schooling choice. Imbens and Newey (2003) motivate a nonparametric nonseparable specification.
} 
There are many situations in which an endogenous variable varies discretely and a nonseparable model is of interest. For example, in an analysis of demand, $Y_{1}$ could be household expenditure on electricity and $Y_{2}$ could measure the number of electrical appliances owned by a household. ${ }^{3}$ In an analysis of firm behaviour, $Y_{1}$ could measure company sales or profits and $Y_{2}$ could measure the number of plants a firm operates, or, in an analysis of innovation, the number of patents registered. ${ }^{4}$

This paper focusses on the case in which an endogenous variable varies discretely. Results for the continuous case, already available, emerge on considering the effect of reducing to zero the distance between the points of support of the discrete endogenous variable. The analysis allows the outcome, $Y_{1}$ and the covariates, $X$, to vary discretely or continuously.

1.3. Related results. Chesher (2002, 2003) and Imbens and Newey $(2003)^{5}$ study identification in nonseparable models of this sort when endogenous variables are continuously distributed. Their results do apply in one case in which endogenous variables vary discretely but this is a case of no econometric interest. ${ }^{6}$

Matzkin (2003) studies identification and estimation of nonseparable structural functions when arguments are exogenous. Roehrig (1988) gives results on global identification in smooth nonseparable models with endogenous variables under continuous variation. Imbens and Newey (2003) relax Roehrig's smoothness restriction and provide estimators and their asymptotic distribution theory for the continuous endogenous variable case. They also study identification and estimation of various average structural functions when there are many sources of stochastic variation.

Chesher $(2002,2003)$ develops local conditions under which there is identification of values, derivatives and partial differences of nonseparable structural functions at specific values of their arguments for the continuous endogenous variable case. That local focus allows relaxation of the restriction requiring latent variates and covariates to be independently distributed, a restriction essential in a global nonparametric identification analysis such as that of Roehrig (1988), Imbens and Newey (2003) and Matzkin (2003).

There is a large literature on nonparametric identification when latent variates are additively separable. See for example Newey, Powell and Vella (1999), Darolles, Florens and Renault (200), Pinkse (2000), Newey and Powell (2003) and Severini and Tripathi (2003) and the references therein.

Nonparametric identification when there are discrete endogenous variables and the structural function is additively separable in the latent variate is studied in Das (2003) and Florens and Malavolti (2003).

\footnotetext{
${ }^{3}$ See Dubin and McFadden (1984) which employs a parametric specification.

${ }^{4}$ See for example Cockburn and Griliches (1988) and the work surveyed in Griliches (1990).

${ }^{5}$ Imbens and Newey (2003) is a revised version of a 2001 manuscript with the same title.

${ }^{6}$ In Chesher $(2002,2003)$ and Imbens and Newey (2003) discrete variation in $Y_{2}$ is only permitted if variation in $X$ affects the values of the points of support of the distribution of $Y_{2}$ but not the probabilities on the points of support.
} 
This paper provides conditions under which there is identification of features of nonseparable structural functions when there are discrete endogenous variables. The outcome and the covariates may be discrete or continuous. The attack on the problem follows the approach taken in Chesher (2003). It is helpful to outline that approach now, as a prelude to describing the method of this paper and presenting its main results.

1.4. Identification with continuous endogenous variables. Extensive use is made of conditional quantile functions for which there is the following notation.

$Q_{A \mid B}(p \mid b)$ denotes the conditional $p$-quantile of scalar random variable $A$ given vector random variable $B=b$. With

$$
F_{A \mid B}(a \mid b) \equiv P[A \leq a \mid B=b]
$$

there is the following definition of $Q_{A \mid B}(p \mid b)$.

$$
Q_{A \mid B}(p \mid b) \equiv \inf \left\{q: F_{A \mid B}(q \mid b) \geq p\right\}
$$

When $Y_{2}$ is continuously distributed, conditions sufficient to identify the value of the structural function $h_{1}$ and its sensitivity to variation in $Y_{2}$ are developed as follows. Substituting for $Y_{2}$ in (1) gives

$$
Y_{1}=h_{1}\left(h_{2}\left(X, \rho_{2}\right), X, \rho_{1}\right)
$$

and because $h_{1}$ is increasing in $\rho_{1}$ there is

$$
Q_{Y_{1} \mid \rho_{2}, X}\left(\tau_{1} \mid \tau_{2}, x\right)=h_{1}\left(h_{2}\left(x, \tau_{2}\right), x, Q_{\rho_{1} \mid \rho_{2}, X}\left(\tau_{1} \mid \tau_{2}, x\right)\right)
$$

because of the equivariance of quantiles under monotone transformation.

When $Y_{2}$ is continuously distributed conditioning on $\rho_{2}=\tau_{2}$ and $X=x$ is identical to conditioning on $Y_{2}=y_{2} \equiv h_{2}\left(x, \tau_{2}\right)$ and $X=x$, and so there is

$$
Q_{Y_{1} \mid Y_{2}, X}\left(\tau_{1} \mid y_{2}, x\right)=h_{1}\left(y_{2}, x, Q_{\rho_{1} \mid \rho_{2}, X}\left(\tau_{1} \mid \tau_{2}, x\right)\right)
$$

It is convenient to normalise $\rho_{2}$ to be uniformly distributed on $(0,1)$ independent of $X$ so that $y_{2} \equiv h_{2}\left(x, \tau_{2}\right)$ is the conditional $\tau_{2}$-quantile of $Y_{2}$ given $X=x$, that is $h_{2}\left(x, \tau_{2}\right)=Q_{Y_{2} \mid X}\left(\tau_{2} \mid x\right)$. With $r_{1}^{*} \equiv Q_{\rho_{1} \mid \rho_{2}, X}\left(\tau_{1} \mid \tau_{2}, x\right)$ equation (3) can then be written as follows.

$$
Q_{Y_{1} \mid Y_{2}, X}\left(\tau_{1} \mid Q_{Y_{2} \mid X}\left(\tau_{2} \mid x\right), x\right)=h_{1}\left(Q_{Y_{2} \mid X}\left(\tau_{2} \mid x\right), x, r_{1}^{*}\right)
$$

The value of the conditional quantile on the left hand side of the equation identifies the value of the structural function on the right hand side.

Suppose that, at some point of interest, $h_{1}$ and $r_{1}^{*}$ are insensitive to some particular variation, $\triangle x$ in $x .^{7}$ If $h_{2}$ is sensitive to this variation there is a nonzero change in

\footnotetext{
${ }^{7}$ Both $x$ and $\triangle x$ may be vectors.
} 
$Q_{Y_{2} \mid X}\left(\tau_{2} \mid x\right)$. Then the value of $Y_{2}$ at which $h_{1}$ is evaluated on the right hand side of (4) is altered as $x$ is moved by $\triangle x$, but $r_{1}^{*}$ remains constant and there is no effect through the $x$ argument of $h_{1}$. The resulting change in the value of the structural function, entirely attributable to variation in the $Y_{2}$ argument, is identified by the effect that moving $x$ by $\triangle x$ has on the conditional quantile on the left hand side of $(4){ }^{8}$

If $X$ can be continuously varied then this argument leads to conditions under which there is identification of partial derivatives of structural function, as described in Chesher (2003). But if $X$ can only vary discretely one must be content with identifying partial differences of structural functions as described in Chesher (2002).

1.5. Discrete endogenous variables. The argument employed in the case in which $Y_{2}$ is continuously distributed cannot be used when $Y_{2}$ is discrete. When $Y_{2}$ varies discretely equation (2) no longer defines a one-to-one relationship between $\left(X, \rho_{2}\right)$ and $\left(X, Y_{2}\right)$ and the crucial step from (3) to (4) cannot be taken.

Let $y_{2}^{m}$ denote the $m$ th of $M$ points of support of the distribution of discrete $Y_{2}$. Fixing $Y_{2}=y_{2}^{m}$ and $X=x$ limits $\rho_{2}$ to the following interval. ${ }^{9}$

$$
\rho_{2} \in\left(F_{Y_{2} \mid X}\left(y_{2}^{m-1} \mid x\right), F_{Y_{2} \mid X}\left(y_{2}^{m} \mid x\right)\right]
$$

The quantile on the left hand side of equation (4) is constant for variations in $\tau_{2}$ in this interval, but $r_{1}^{*} \equiv Q_{\rho_{1} \mid \rho_{2}, X}\left(\tau_{1} \mid \tau_{2}, x\right)$ which appears as an argument of the right hand side of (4) varies as $\tau_{2}$ is altered or $Y_{2}$ is locally exogenous. So, when $Y_{2}$ is discrete and at least locally endogenous equation (4) cannot hold.

Under weak nonparametric restrictions the value of a structural function at specific values of its arguments cannot be point identified when one of the arguments is a discrete endogenous variable. Without point identification of the value of a structural function there is no hope of point identification of the effect on the structural function of varying an endogenous, or indeed an exogenous, argument.

1.6. Set identification. The fact that fixing $Y_{2}$ and $X$ restricts $\rho_{2}$ to an interval hints at the possibility of set identification. Define

$$
r_{1}^{*} \equiv Q_{\rho_{1} \mid \rho_{2} X}\left(\tau_{1} \mid \tau_{2}, x\right)
$$

and

$$
p^{m}(x) \equiv F_{Y_{2} \mid X}\left(y_{2}^{m} \mid x\right) .
$$

Theorem 1, given in Section 2, states that if, for some point of support $y_{2}^{m}$ and probability $\tau_{2}$, there exist values of $X, x^{m-1}$ and $x^{m}$ such that

$$
p^{m}\left(x^{m}\right) \leq \tau_{2} \leq p^{m-1}\left(x^{m-1}\right)
$$

\footnotetext{
${ }^{8}$ Note that on the left hand side of $(4)$ changing $x$ by $\triangle x$ affects $Q_{Y_{2} \mid X}\left(\tau_{2} \mid x\right)$, and thus the $Y_{2}$ argument of $Q_{Y_{1} \mid Y_{2} X}$ and also the $X$ argument of $Q_{Y_{1} \mid Y_{2} X}$.

${ }^{9}$ To cover the case $m=1, F_{Y_{2} \mid X}\left(y_{2}^{0} \mid x\right)$ is defined to be zero for all $x$, and note that $F_{Y_{2} \mid X}\left(y_{2}^{M} \mid x\right)=$ 1.
} 
then under certain conditions and for $x \in\left\{x^{m-1}, x^{m}\right\}$, there is the following interval identifying inequality.

$$
\begin{gathered}
\min \left(Q_{Y_{1} \mid Y_{2} X}\left(\tau_{1} \mid y_{2}^{m}, x^{m-1}\right), Q_{Y_{1} \mid Y_{2} X}\left(\tau_{1} \mid y_{2}^{m}, x^{m}\right)\right) \\
\quad \leq h_{1}\left(y_{2}^{m}, x, r_{1}^{*}\right) \leq \\
\max \left(Q_{Y_{1} \mid Y_{2} X}\left(\tau_{1} \mid y_{2}^{m}, x^{m-1}\right), Q_{Y_{1} \mid Y_{2} X}\left(\tau_{1} \mid y_{2}^{m}, x^{m}\right)\right)
\end{gathered}
$$

The conditions under which (6) holds include the following.

1. For $r_{1}$ in a sufficiently wide interval around $r_{1}^{*}, F_{\rho_{1} \mid \rho_{2} X}\left(r_{1} \mid r_{2}, x\right)$ is monotonic in $r_{2}$ for $r_{2}$ satisfying the following inequality.

$$
p^{m-1}\left(x^{m}\right) \leq r_{2} \leq p^{m}\left(x^{m-1}\right)
$$

2. The value of the structural function $h_{1}\left(y_{2}^{m}, x, r_{1}^{*}\right)$ is insensitive to variation in $x$ $\in\left\{x^{m-1}, x^{m}\right\}$.

3. The value $r_{1}^{*}$ is insensitive to variation in $x \in\left\{x^{m-1}, x^{m}\right\}$.

The inequality (6) does not apply when $y_{2}^{m}$ is an extreme point of support, so the binary endogenous variable case is excluded from consideration. When $y_{2}^{m}$ is the lowest (respectively highest) point of support of $Y_{2}$ the probability on the right (respectively left) of (5) is zero (respectively one). Then (5) could only be satisfied if $\tau_{2}$ were zero (respectively one) but then the probability mass at $y_{2}^{m}$ would be zero and the object whose identification is sought vanishes.

The argument leading to the interval identification result is now sketched. The seminal work of Hurwicz (1950) makes clear that in any study of identification one must consider how the information contained in the conditional distribution of outcomes $\left(Y_{1}\right.$ and $\left.Y_{2}\right)$ given covariates $(X)$ bears on the structural features whose identification is sought Thus the route to the interval identification result (6) starts with consideration of the conditional distribution of $Y_{1}$ given $Y_{2}=y_{2}^{m}$ and $X=x$ and the way in which this is related to the structural function evaluated at $y_{2}^{m}$ and $x$.

Lemma 2 in the Appendix to the paper provides bounds on this conditional distribution function and its associated quantile function. The bounds on the quantile function are as follows.

$$
\begin{gathered}
h^{L}\left(\tau_{1}, y_{2}^{m}, x\right) \equiv \min \left\{\begin{array}{c}
h_{1}\left(y_{2}^{m}, x, Q_{\rho_{1} \mid \rho_{2} X}\left(\tau_{1} \mid p^{m-1}(x), x\right)\right) \\
h_{1}\left(y_{2}^{m}, x, Q_{\rho_{1} \mid \rho_{2} X}\left(\tau_{1} \mid p^{m}(x), x\right)\right)
\end{array}\right\} \\
\leq Q_{Y_{1} \mid Y_{2} X}\left(\tau_{1} \mid y_{2}^{m}, x\right) \leq \\
\max \left\{\begin{array}{c}
h_{1}\left(y_{2}^{m}, x, Q_{\rho_{1} \mid \rho_{2} X}\left(\tau_{1} \mid p^{m-1}(x), x\right)\right) \\
h_{1}\left(y_{2}^{m}, x, Q_{\rho_{1} \mid \rho_{2} X}\left(\tau_{1} \mid p^{m}(x), x\right)\right)
\end{array}\right\} \equiv h^{U}\left(\tau_{1}, y_{2}^{m}, x\right)
\end{gathered}
$$

Suppose $Q_{Y_{1} \mid Y_{2} X}\left(\tau_{1} \mid y_{2}^{m}, x^{m}\right) \geq Q_{Y_{1} \mid Y_{2} X}\left(\tau_{1} \mid y_{2}^{m}, x^{m-1}\right)$. Under the conditions stated above the lower bound on $Q_{Y_{1} \mid Y_{2} X}\left(\tau_{1} \mid y_{2}^{m}, x^{m}\right)$, is no smaller than the upper bound 
on $Q_{Y_{1} \mid Y_{2} X}\left(\tau_{1} \mid y_{2}^{m}, x^{m-1}\right)$ and $h_{1}\left(y_{2}^{m}, x, r_{1}^{*}\right)$ lies between these bounds, that is, for $x$ $\in\left\{x^{m-1}, x^{m}\right\}$ :

$$
h^{L}\left(\tau_{1}, y_{2}^{m}, x^{m}\right) \geq h_{1}\left(y_{2}^{m}, x, r_{1}^{*}\right) \geq h^{U}\left(\tau_{1}, y_{2}^{m}, x^{m-1}\right) .
$$

The two quantile functions therefore bound $h_{1}\left(y_{2}^{m}, x, r_{1}^{*}\right)$. When $Q_{Y_{1} \mid Y_{2} X}\left(\tau_{1} \mid y_{2}^{m}, x^{m}\right) \leq$ $Q_{Y_{1} \mid Y_{2} X}\left(\tau_{1} \mid y_{2}^{m}, x^{m-1}\right)$ the inequalities are reversed and combining the results leads to the interval identifying inequality (6).

1.7. Main results and the organisation of the paper. The precise results concerning identification of the value of a structural function are given in Section 2. In many cases identification of the sensitivity of a structural function to changes in an endogenous variable is of primary interest. Identification of structural values is treated first because this is an essential prerequisite for identification of structural differences.

In Section 2 Theorem 1 defines a model and gives a result which is shown to imply that the model interval identifies the value of a structural function of a discrete endogenous variable at specific values of its arguments.

Theorem 2 shows that the interval identifying bounds are sharp in the sense that there can exist admissible structures such that the bounds are attained. For example the bounds are attained when $\rho_{1}$ and $\rho_{2}$ are distributed independently given $X$, in which case $Y_{2}$ is exogenous.

Theorem 3 implies that if $\rho_{1}$ and $\rho_{2}$ co-vary more than minimally then point identification of the value of a structural function is not feasible if $Y_{1}$ is continuously distributed and $Y_{2}$ is discrete. In that situation there are no values, $x^{m-1}$ and $x^{m}$, such that

$$
Q_{Y_{1} \mid Y_{2} X}\left(\tau_{1} \mid y_{2}^{m}, x^{m-1}\right)-Q_{Y_{1} \mid Y_{2} X}\left(\tau_{1} \mid y_{2}^{m}, x^{m}\right)=0
$$

so the interval (6) always has non-zero length.

Analogue estimation of identifying intervals and the issue of determining minimum length intervals are discussed in Section 2.7.

If $y_{2}^{m}$ is very close to $y_{2}^{m-1}$, which happens when $Y_{2}$ is close to being continuously distributed, then it may be possible to find $x^{m-1}$ and $x^{m}$ such that (7) is "nearly" satisfied and the identifying interval is short. Considering the limiting case, if $Y_{2}$ is continuously distributed and there exists $x^{*}$ such that $\tau_{2}=F_{Y_{2} \mid X}\left(y_{2}^{*} \mid x^{*}\right)$, and therefore $y_{2}^{*} \equiv Q_{Y_{2} \mid X}\left(\tau_{2} \mid x^{*}\right)$, then there is point identification of $h_{1}\left(y_{2}^{*}, x, r_{1}\right)$, as described in Section 1.4. This is the subject of Section 2.8.

Section 3 gives results on the identification of structural partial differences. Identification of a partial difference can be achieved if each component of the difference is interval identified and if the structural function and the value of its $\rho_{1}$ argument are insensitive to the variations in $X$ required to identify the components. Section 3.1 and 3.2 deal with interval identification of a structural partial difference with respect to respectively an endogenous variate and a covariate.

Section 4 examines the implications of these results for the well known study of Angrist and Krueger (1991) (AK) which uses wage information and discrete data on 
years of schooling to estimate returns to schooling. Analysis of the AK data suggests that the weak quarter of birth instruments have insufficient power to identify the returns to schooling in the absence of parametric restrictions of the sort imposed by AK. ${ }^{10}$ Results are presented suggesting that, even if schooling were continuously recorded, the weakness and discreteness of the quarter of birth instruments severely limits the structural information that can be extracted from this data without imposing the sort of parametric restrictions used by AK.

Section 5 concludes with a discussion of the merits of local identification analysis, the extension of the analysis to cases in which there are many endogenous variables, the issue of identification when an endogenous variate is binary, and the relationship of this analysis to the classical Cowles Commission analysis of identification.

\section{Identification of a VAlue Delivered By a StruCtural FUnCtion}

2.1. Introduction and definitions. This Section considers nonparametric identification of the value of a discrete or continuous random variable $Y_{1}$ delivered by a structural function when one of the arguments of the structural function, $Y_{2}$, is a discrete endogenous variable. Identification of structural partial differences is addressed in Section 3.

First there are the following definitions.

Definition 1. $X \equiv\left\{X_{k}\right\}_{k=1}^{K}$ is a vector of covariates. The conditional distribution and quantile functions of $\rho_{1}$ given $\rho_{2}$ and $X$ are denoted by respectively $F_{\rho_{1} \mid \rho_{2} X}$ and $Q_{\rho_{1} \mid \rho_{2} X} \cdot \rho_{1}$ and $\rho_{2}$ are jointly continuously distributed latent variates and $\rho_{2}$ is normalised uniformly distributed on $(0,1)$ independent of $X$.

Definition 2. $Y_{1}$ is a discrete, continuous or mixed discrete-continuous random variable. The conditional distribution of $Y_{2}$ given $X=x$ is discrete with countable points of support $\left\{y_{2}^{m}\right\}_{m=1}^{M}$, invariant with respect to $x$, and with positive probability masses $\left\{p_{m}(x)\right\}_{m=1}^{M}$. Cumulative probabilities $\left\{p^{m}(x)\right\}_{m=1}^{M}$ are defined as:

$$
p^{m}(x) \equiv \sum_{l=0}^{m} p_{l}(x), \quad m \in\{1, \ldots, M\}
$$

with $p^{0}(x) \equiv 0$ and $p^{M}(x)=1$.

Restriction A1 imposes conditions on the structural functions determining $Y_{1}$ and $Y_{2}$ and will be maintained throughout.

(A1). Structural functions. At any value of $X, \rho_{1}$ and $\rho_{2}$, the value of the outcomes $Y_{1}$ and $Y_{2}$ is uniquely determined by the following equations.

$$
\begin{aligned}
& Y_{1}=h_{1}\left(Y_{2}, X, \rho_{1}\right) \\
& Y_{2}=h_{2}\left(X, \rho_{2}\right)
\end{aligned}
$$

\footnotetext{
${ }^{10}$ This is additional to the problem weak instruments pose for inference discussed in Bound, Jaeger and Baker (1995).
} 
The function $h_{1}$ is weakly monotonic with respect to variation in $\rho_{1}$, normalised caglad and non-decreasing. The function $h_{2}$ evaluated at $X=x, h_{2}\left(x, \rho_{2}\right)$ is $Q_{Y_{2} \mid X}\left(\rho_{2} \mid x\right)$, the conditional quantile functionof $Y_{2}$ given $X=x$, as follows.

$$
h_{2}\left(x, \rho_{2}\right)=\left\{\begin{array}{ll}
y_{2}^{1} & p^{0}(x)=0<\rho_{2} \leq p^{1}(x) \\
y_{2}^{m} & p^{m-1}(x)<\rho_{2} \leq p^{m}(x), \\
y_{2}^{M} & p^{M-1}(x)<\rho_{2} \leq p^{M}(x)=1
\end{array} \quad m \in\{2, \ldots, M-1\}\right.
$$

The structural equations (8) and (9) are in triangular form. This is essential in what follows but is less restrictive than it seems at first sight. The second equation can be regarded as a "reduced form" equation in a fully simultaneous structural system, so the analysis which follows can be regarded as an analysis of the identification of features of a single structural equation embedded in a fully simultaneous system.

2.2. Identifying restrictions. This Section provides nonparametric restrictions under which there is identification of the value, $h_{1}\left(y_{2}^{m}, x, r_{1}^{*}\right)$, delivered by the structural function $h_{1}$. The value $y_{2}^{m}, m \in\{2, \ldots, M-1\}$, is an interior point of support of the distribution of $Y_{2}$. The term $r_{1}^{*}$ is a value of $\rho_{1}$ defined as

$$
r_{1}^{*} \equiv Q_{\rho_{1} \mid \rho_{2} X}\left(\tau_{1} \mid \tau_{2}, x\right)
$$

where $\tau \equiv\left\{\tau_{1}, \tau_{2}\right\} \in(0,1)^{2}$ and $x$ lies in a set of instrumental values of $X$ to be specified shortly. ${ }^{11}$

Four restrictions additional to $\mathrm{A} 1$ are now introduced. These define a set, $\tilde{x}_{m}$, of instrumental values of $X$ and ensure that $r_{1}^{*}$ and $h_{1}\left(y_{2}^{m}, x, r_{1}^{*}\right)$ are invariant with respect to choice of $x$ within the set $\tilde{x}_{m}$.

(B1) There exist instrumental values of $X, \tilde{x}_{m} \equiv\left\{x^{m-1}, x^{m}\right\}$, such that

$$
p^{m}\left(x^{m}\right) \equiv F_{Y_{2} \mid X}\left(y_{2}^{m} \mid x^{m}\right) \leq \tau_{2} \leq F_{Y_{2} \mid X}\left(y_{2}^{m-1} \mid x^{m-1}\right) \equiv p^{m-1}\left(x^{m-1}\right) .
$$

(B2) For $x \in \tilde{x}_{m}$ and all $r_{1}$, and $r_{2} \in R_{2} \equiv\left(p^{m-1}\left(x^{m}\right), p^{m}\left(x^{m-1}\right)\right], F_{\rho_{1} \rho_{2} X}\left(\tau_{1} \mid r_{2}, x\right)$ is a monotonic function of $r_{2}$.

(B3) The value of $r_{1}^{*} \equiv Q_{\rho_{1} \mid \rho_{2} X}\left(\tau_{1} \mid \tau_{2}, x\right)$ is invariant with respect to choice of $x \in$ $\tilde{x}_{m}$.

(B4) The value of $h_{1}\left(y_{2}^{m}, x, r_{1}^{*}\right)$ is invariant with respect to choice of $x \in \tilde{x}_{m}$, that is:

$$
h_{1}\left(y_{2}^{m}, x^{m}, r_{1}^{*}\right)=h_{1}\left(y_{2}^{m}, x^{m-1}, r_{1}^{*}\right) .
$$

\footnotetext{
${ }^{11}$ In a classical analysis of identifcation, one identifies a subset of the covariates as instrumental variables. In this analysis of local identification it is allowed that variables can play the classical role of instrumental variables at some values but not at others. Hence the term instrumental values is used.
} 
2.3. Remarks on the restrictions. Restrictions B1 and B4 taken together are analogous to the rank condition in the classical analysis of identification ${ }^{12}$ of the values of parameters of a linear structural equation, Restriction B1 requiring the distribution of $Y_{2}$ given $X$ to have a degree of sensitivity to variation in $X$, Restriction B4 limiting the sensitivity of the structural function to variation in $X$

It follows directly from (11) that

$$
Q_{Y_{2} \mid X}\left(\tau_{2} \mid x^{m-1}\right) \leq y_{2}^{m-1}<y_{2}^{m} \leq Q_{Y_{2} \mid X}\left(\tau_{2} \mid x^{m}\right)
$$

the strong inequality, $y_{2}^{m-1}<y_{2}^{m}$, holding because $Y_{2}$ has a discrete distribution with positive probability masses on each point of support. So Restriction B1 requires $X$ to have impact on the conditional distribution of $Y_{2}$ given $X$ sufficient that both $y_{2}^{m}$ and $y_{2}^{m-1}$ are bounded by $\tau_{2}$-quantiles of the distribution of $Y_{2}$ given $X$ and that there exist values of $X$ such that this bound can be achieved.

If $\left\{x^{m-1}, x^{m}\right\}$ are such that equalities hold throughout in (11) then $y_{2}^{m}$ and $y_{2}^{m-1}$ are $\tau_{2}$-quantiles of $Y_{2}$ given $X$ equal to respectively $x^{m}$ and $x^{m-1}$. Such pairs of values of $X$ may exist if $X$ has rich support, for example if $X$ is continuously distributed, and the impact of $x$ on $Q_{Y_{2} \mid X}\left(\tau_{2} \mid x\right)$ is sufficiently strong.

Restriction B4 is a weak "local exclusion" restriction requiring insensitivity of the structural function $h_{1}$ to variation in $X$ within $\tilde{x}_{m}$ at the point of interest. It would hold if $X$ were entirely excluded from $h_{1}$. It would hold if certain elements of $X$ were excluded from $h_{1}$, in which case the non-excluded elements would be required to be equal in $x^{m-1}$ and $x^{m}$. Restriction B4 is rather weak, requiring only local insensitivity of $h_{1}$ to variation in $X$.

The essential requirement covered by Restriction B2 is that $F_{\rho_{1} \rho_{2} X}$, and therefore $Q_{\rho_{1} \rho_{2} X}$, have a degree of bounded variation with respect to $\rho_{2}$ sufficient to allow the impact of $\rho_{2}$ on $h_{1}$ (via the dependence of $\rho_{1}$ on $\rho_{2}$ ) to be limited when $Y_{2}=y_{2}^{m}$. The monotonicity restriction of B2 is sufficient for this purpose and can be slightly weakened.

Restriction B3 limits the dependence of $\rho_{1}$ on $X$ given $\rho_{2}$. It would hold if $\rho_{1}$ were distributed independently of $X$ given $\rho_{2}$ but is much weaker being local to $\tau$ and to the values of $x$ in $\tilde{x}_{m}$.

Identification of $h_{1}\left(y_{2}^{m}, x, r_{1}^{*}\right)$ when $y_{2}^{m}$ is the lowest or highest point of support of $Y_{2}$ is excluded from consideration. Thus the identification results developed here have no force when $Y_{2}$ is binary.

Restriction B1, which plays a key role in this nonparametric analysis, cannot be satisfied in either of these cases. Consider the case $m=M$, so that $y_{2}^{m}$ is the maximum value of $Y_{2}$. The cumulative probability $p^{M}\left(x^{M}\right)$ must be equal to one for all $x^{M}$ and there can be no value of $\tau_{2} \in(0,1)$ such that $\tau_{2} \geq p^{M}\left(x^{M}\right)=1$. If $\tau_{2}=1$ were admitted then Restriction B1 could only be satisfied for $x^{M-1}$ such that $p^{M-1}\left(x^{M-1}\right)=1$ but then there is no probability mass on $y_{2}^{M}$ which violates the requirement that all points of support of $Y_{2}$ carry positive probability.

\footnotetext{
${ }^{12}$ Koopmans, Rubin and Leipnik (1950).
} 
Consider the case $m=1$, so that $y_{2}^{m}$ is the minimum value of $Y_{2}$. The cumulative probability $p^{0}(x)=0$ for all $x$ so Restriction B1 requires $\tau_{2} \leq 0$ which cannot be satisfied if $\tau_{2} \in(0,1)$. If $\tau_{2}=0$ were admitted then Restriction B1 requires $x^{1}$ to be chosen such that $p^{1}\left(x^{1}\right)=0$ but then there is no probability mass on $y_{2}^{1}$ which violates the requirement that all points of support of $Y_{2}$ carry positive probability.

The latent variate $\rho_{2}$ is not an argument of the structural function $h_{1}$. Chesher (2002, 2003) and Imbens and Newey (2003), both effectively considering the case in which $Y_{2}$ is continuously distributed, demonstrate identification of the value of a structural function when $\rho_{1}$ and $\rho_{2}$ are arguments of $h_{1}$. This case can be addressed using the methods of this paper if Restriction B2 is modified. The case in which the structural function (8) has the form

$$
Y_{1}=h_{1}\left(Y_{2}, X, \rho_{1}, \rho_{2}\right)
$$

can be addressed if Restriction B2 is replaced by the following restriction.

$$
\begin{aligned}
& \text { For } x \in \tilde{x}_{m} \text { and all } t_{1} \text {, and } r_{2} \in R_{2} \equiv\left(p^{m-1}\left(x^{m}\right), p^{m}\left(x^{m-1}\right)\right] \\
& \qquad h_{1}\left(y_{2}^{m}, x, Q_{\rho_{1} \rho_{2} X}\left(t_{1} \mid r_{2}, x\right), r_{2}\right)
\end{aligned}
$$

is a monotonic function of $r_{2}$.

The results which follow apply with this modification. Like Restriction B2, B2 ${ }^{\S}$ can be weakened to a local (to $\tau_{1}$ ) bounded variation restriction.

2.4. Set identification. Hurwicz (1950) defines the concept of point identification of a feature of a structure. The definition is extended to cover set identification as follows.

Definition 3. A model identifies the value of a feature of a structure $S_{0}$ within a set $A_{0}$ if in all structures admitted by the model and observationally equivalent to $S_{0}$ the value of the feature lies in $A_{0}$.

The model defined by the restrictions given in Section 2.2 set identifies $h_{1}\left(y_{2}^{m}, x, r_{1}^{*}\right)$. This is the subject of Theorem 1. Define

$$
\begin{aligned}
q^{L}\left(\tau, y_{2}^{m}, \tilde{x}_{m}\right) & \equiv \min \left(Q_{Y_{1} \mid Y_{2} X}\left(\tau_{1} \mid y_{2}^{m}, x^{m-1}\right), Q_{Y_{1} \mid Y_{2} X}\left(\tau_{1} \mid y_{2}^{m}, x^{m}\right)\right) \\
q^{U}\left(\tau, y_{2}^{m}, \tilde{x}_{m}\right) & \equiv \max \left(Q_{Y_{1} \mid Y_{2} X}\left(\tau_{1} \mid y_{2}^{m}, x^{m-1}\right), Q_{Y_{1} \mid Y_{2} X}\left(\tau_{1} \mid y_{2}^{m}, x^{m}\right)\right)
\end{aligned}
$$

and recall the definition (10) of $r_{1}^{*}$.

Theorem 1. Under Restrictions A1 and B1 - B4 there is the inequality:

$$
q^{L}\left(\tau, y_{2}^{m}, \tilde{x}_{m}\right) \leq h_{1}\left(y_{2}^{m}, x, r_{1}^{*}\right) \leq q^{U}\left(\tau, y_{2}^{m}, \tilde{x}_{m}\right)
$$

where $x \in \tilde{x}_{m} \equiv\left\{x^{m-1}, x^{m}\right\}$. 
The proof is in the Appendix. The following argument shows that Theorem 1 implies set identification of the value of $h_{1}\left(y_{2}^{m}, x, r_{1}^{*}\right)$.

The objects $q^{L}\left(\tau, y_{2}^{m}, \tilde{x}_{m}\right)$ and $q^{U}\left(\tau, y_{2}^{m}, \tilde{x}_{m}\right)$ are well-defined, single valued functionals of the distribution of $Y_{1}$ and $Y_{2}$ given $X$. Consider any admissible structure, $S$, that is a structure satisfying Restrictions A1 and B1 - B4, in which $h_{1}\left(y_{2}^{m}, x, r_{1}^{*}\right)=h^{S}$. Theorem 1 states that

$$
q_{S}^{L} \leq h_{1}^{S} \leq q_{S}^{U}
$$

where $q_{S}^{L}$ and $q_{S}^{U}$ are the values obtained when the functionals (13) and (14) are applied to the distribution of $Y_{1}$ and $Y_{2}$ given $X$ generated by the structure $S$. Consider any admissible structure $S^{\prime}$ observationally equivalent to $S$ with $h_{1}\left(y_{2}^{m}, x, r_{1}^{*}\right)=h^{S^{\prime}}$. Since $S^{\prime}$ is observationally equivalent to $S$ it generates the same distribution for $Y_{1}$ and $Y_{2}$ given $X$ as does the structure $S$, and so it yields the same values of $q^{L}$ and $q^{U}$, that is $q_{S}^{L}=q_{S^{\prime}}^{L}$ and $q_{S}^{U}=q_{S^{\prime}}^{U}$ and Theorem 1 tells us that

$$
q_{S}^{L} \leq h_{1}^{S^{\prime}} \leq q_{S}^{U}
$$

whatever the value of $h_{1}^{S^{\prime}}$. Theorem 1 therefore implies that the model defined by the restrictions of Theorem 1 set identifies the value of $h_{1}\left(y_{2}^{m}, x, r_{1}^{*}\right)$ in the sense of Definition 3.

2.5. Sharpness of the bounds and locally exogenous $Y_{2}$. The bounds of Theorem 1 are sharp in the sense that there can exist admissible structures in which equalities hold throughout in (15). One such case is the subject of Theorem 2 . Consider the following restriction in place of Restriction B2.

$\left(\mathrm{B} 2^{*}\right)$ For each $x \in \tilde{x}_{m}$ and all $r_{1}, F_{\rho_{1} \rho_{2} X}\left(r_{1} \mid r_{2}, x\right)$ is constant for variation in $r_{2}$ in $R_{2} \equiv\left(p^{m-1}\left(x^{m}\right), p^{m}\left(x^{m-1}\right)\right]$.

Theorem 2. Under Restrictions A1, B1, B2*, B3 and B4 there is the equality

$$
h_{1}\left(y_{2}^{m}, x, r_{1}^{*}\right)=Q_{Y_{1} \mid Y_{2} X}\left(\tau_{1} \mid y_{2}^{m}, x^{m}\right)=Q_{Y_{1} \mid Y_{2} X}\left(\tau_{1} \mid y_{2}^{m}, x^{m-1}\right)
$$

where $x \in \tilde{x}_{m}$.

Theorem 2 not only demonstrates the sharpness of the bounds of Theorem 1, but also shows that when $Y_{2}$ is "locally exogenous" point identification of $h_{1}\left(y_{2}^{m}, x, r_{1}^{*}\right)$ is possible if suitable instrumental values can be found. ${ }^{13}$

\footnotetext{
${ }^{13}$ This result was given in Chesher (2002).
} 
2.6. Point and interval identification. Theorem 1 implies that the value of $h_{1}\left(y_{2}^{m}, x, r_{1}^{*}\right)$ is point identified if:

$$
Q_{Y_{1} \mid Y_{2} X}\left(\tau_{1} \mid y_{2}^{m}, x^{m-1}\right)=Q_{Y_{1} \mid Y_{2} X}\left(\tau_{1} \mid y_{2}^{m}, x^{m}\right) .
$$

When $Y_{1}$ is a discrete random variable this equality can hold because the discretely varying quantiles of $Y_{1}$ given $Y_{2}$ and $X$ can be insensitive to variation in $x \in \tilde{x}_{m}$.

When $Y_{1}$ is continuously distributed the equality (16) cannot hold unless the conditions of Theorem 2 prevail. This is the subject of Theorem 3. Consider the following restriction in place of Restriction B2.

$\left(\mathrm{B} 2^{* *}\right)$ For each $x \in \tilde{x}_{m}$ and all $r_{1}, F_{\rho_{1} \rho_{2} \mid X}\left(r_{1} \mid r_{2}, x\right)$ is monotonic and non-constant for variation in $r_{2}$ in $R_{2} \equiv\left(p^{m-1}\left(x^{m}\right), p^{m}\left(x^{m-1}\right)\right]$. $h_{1}$ is strictly montonic in $\rho_{1}$.

Theorem 3. Under Restrictions A1, B1, B2**, B3 and B4

$$
Q_{Y_{1} \mid Y_{2} X}\left(\tau_{1} \mid y_{2}^{m}, x^{m-1}\right) \neq Q_{Y_{1} \mid Y_{2} X}\left(\tau_{1} \mid y_{2}^{m}, x^{m}\right) .
$$

and $h_{1}\left(y_{2}^{m}, x, r_{1}^{*}\right)$ is not point identified.

An implication of Theorem 3 is that when $Y_{1}$ is continuous and $Y_{2}$ is discrete, the model defined by the restrictions of the Theorem 1 only interval identifies the value of $h_{1}\left(y_{2}^{m}, x, r_{1}^{*}\right)$.

There may be many values $\left\{x^{m-1}, x^{m}\right\}$ for which the conditions of Theorem 1 are satisfied and so many identifying intervals. Clearly the interval with minimum length is obtained using $\left\{x_{o p t}^{m-1}, x_{o p t}^{m}\right\}$ defined as:

$$
\left\{x_{o p t}^{m-1}, x_{o p t}^{m}\right\}=\underset{\left\{x^{m-1}, x^{m}\right\} \in B}{\arg \min }\left|Q_{Y_{1} \mid Y_{2} X}\left(\tau_{1} \mid y_{2}^{m}, x^{m-1}\right)-Q_{Y_{1} \mid Y_{2} X}\left(\tau_{1} \mid y_{2}^{m}, x^{m}\right)\right|
$$

where $B$ is the set of values of $X$ for which Restrictions B1 - B4 hold.

There is interval overidentification of $h_{1}\left(y_{2}^{m}, x, r_{1}^{*}\right)$ if there are many pairs $\tilde{x}_{m}$ satisfying the conditions of Theorem 1 leading to the same interval (15). Such a situation can arise, for example, when $X$ contains many elements with more than one of them "excluded" from the structural function $h_{1}$.

The support of $X$ may limit the values of $Y_{2}$ and $\tau_{1}$ at which interval identification can be obtained. Even if there is rich support, when instruments $(X)$ are weak, having only a small influence on the conditional distribution of $Y_{2}$ given $X$, it may not be possible to find values of $X$ satisfying (11) for any value of $\tau_{2}$. The combination of weak instruments and sparse support can combine to produce underidentification at all values of $Y_{2}, \tau_{1}$ and $\tau_{2}$, as is illustrated in Section 4 using data employed in the study of the returns to schooling reported in Angrist and Krueger (1991). 
2.7. Estimation. Identifying intervals can be estimated by applying the analog principle, employing non-, semi- or fully- parametric estimates of the conditional distribution or quantile functions of $Y_{2}$ given $X$ and of $Y_{1}$ given $Y_{2}$ and $X{ }^{14}$

Given a value, $y_{2}^{m}$ of interest, a value $\tau_{2}$ and an estimate $\hat{F}_{Y_{2} \mid X}$ of the conditional distribution function of $Y_{2}$ given $X$ one seeks values $\hat{x}^{m-1}$ and $\hat{x}^{m}$ such that

$$
\hat{F}_{Y_{2} \mid X}\left(y_{2}^{m} \mid \hat{x}^{m}\right) \leq \tau_{2} \leq \hat{F}_{Y_{2} \mid X}\left(y_{2}^{m-1} \mid \hat{x}^{m-1}\right)
$$

and calculates the values of an estimate of the conditional $\tau_{1}$-quantile function of $Y_{1}$ given $Y_{2}=y_{2}^{m}$ and $X=x$ with $x \in\left\{\hat{x}^{m-1}, \hat{x}^{m}\right\}$. This procedure delivers an estimated interval

$$
\left[\hat{q}^{L}\left(\tau, y_{2}^{m}, \widehat{\tilde{x}_{m}}\right), \hat{q}^{U}\left(\tau, y_{2}^{m}, \widehat{\tilde{x}_{m}}\right)\right] .
$$

A minimum length interval can be calculated using $\left\{\hat{x}_{o p t}^{m-1}, \hat{x}_{o p t}^{m}\right\}$ obtained as

$$
\left\{\hat{x}_{o p t}^{m-1}, \hat{x}_{o p t}^{m}\right\}=\underset{\left\{x^{m-1}, x^{m}\right\} \in B \cap \hat{C}}{\arg \min }\left|\hat{Q}_{Y_{1} \mid Y_{2} X}\left(\tau_{1} \mid y_{2}^{m}, x^{m-1}\right)-\hat{Q}_{Y_{1} \mid Y_{2} X}\left(\tau_{1} \mid y_{2}^{m}, x^{m}\right)\right|
$$

where, as above $B$ defines the set of values of $X$ satisfying Restrictions B1 - B4 and

$$
\hat{C} \equiv\left\{\left(x^{m-1}, x^{m}\right): \hat{F}_{Y_{2} \mid X}\left(y_{2}^{m} \mid x^{m}\right) \leq \tau_{2} \leq \hat{F}_{Y_{2} \mid X}\left(y_{2}^{m-1} \mid x^{m-1}\right)\right\} .
$$

This throws up challenging inferential issues, not addressed here. ${ }^{15}$

2.8. Continuous endogenous variables. If $Y_{2}$ is continuously distributed then in (11) we can set $y_{2}^{m}=y_{2}^{m-1}=y_{2}^{*}$ say. The monotonicity restriction B2 is then irrelevant. Suppose there exist $\left\{x^{\prime}, x^{\prime \prime}\right\}$ such that

$$
F_{Y_{2} \mid X}\left(y_{2}^{*} \mid x^{\prime}\right) \leq \tau_{2} \leq F_{Y_{2} \mid X}\left(y_{2}^{*} \mid x^{\prime \prime}\right)
$$

and that $r_{1}^{*} \equiv Q_{\rho_{1} \mid \rho_{2} X}\left(\tau_{1} \mid \tau_{2}, x\right)$ and $h_{1}\left(y_{2}^{*}, x, r_{1}^{*}\right)$ are invariant with respect to choice of $x \in\left\{x^{\prime}, x^{\prime \prime}\right\}$. Then the argument employed in the proof of Theorem 1 leads to the identifying inequality

$$
\begin{gathered}
\min \left(Q_{Y_{1} \mid Y_{2} X}\left(\tau_{1} \mid y_{2}^{*}, x^{\prime}\right), Q_{Y_{1} \mid Y_{2} X}\left(\tau_{1} \mid y_{2}^{*}, x^{\prime \prime}\right)\right) \\
\leq h_{1}\left(y_{2}^{*}, x, r_{1}^{*}\right) \leq \\
\max \left(Q_{Y_{1} \mid Y_{2} X}\left(\tau_{1} \mid y_{2}^{*}, x^{\prime}\right), Q_{Y_{1} \mid Y_{2} X}\left(\tau_{1} \mid y_{2}^{*}, x^{\prime \prime}\right)\right)
\end{gathered}
$$

\footnotetext{
${ }^{14}$ For the case in which $Y_{1}$ is continuous see Koenker and Bassett (1978), Koenker and d'Orey (1987) and Ma and Koenker (2003) for parametric estimation, see Chaudhuri, Doksum and Samarov (1997), Kahn (2001) and Lee (2003a, 2003b) for semi-parametric estimation and see Chaudhuri (1991) for nonparametric estimation. There are fewer options for the case in which $Y_{1}$ is discrete. Machado and Santos Silva (2002) propose a procedure for the parametric case. One could proceed by estimating the conditional distribution function of $Y_{1}$ given $Y_{2}$ and $X$, and obtain an estimated quantile function by inversion.

${ }^{15}$ See Chernozhukov, Hong and Tamer (2003) and Imbens and Manski (2003) for results on inference when there is interval identification of structural features.
} 
where $x \in\left\{x^{\prime}, x^{\prime \prime}\right\}$.

When $X$ has rich support there may exist $x^{*}$ such that $F_{Y_{2} \mid X}\left(y_{2}^{*} \mid x^{*}\right)=\tau_{2}$ and setting $x^{\prime}=x^{\prime \prime}=x^{*}$ in (18) leads to the point identifying equality

$$
h_{1}\left(y_{2}^{*}, x^{*}, r_{1}^{*}\right)=Q_{Y_{1} \mid Y_{2} X}\left(\tau_{1} \mid y_{2}^{*}, x^{*}\right)
$$

where $y_{2}^{*} \equiv Q_{Y_{2} X}\left(\tau_{2} \mid x^{*}\right){ }^{16}$

\section{IDENTIFICATION OF STRUCTURAL PARTIAL DIFFERENCES}

3.1. Identification of a partial difference with respect to an endogenous variable. This Section provides conditions under which there is identification of the value of the structural partial difference $\triangle_{i j} h_{1}^{*}$ defined as follows.

$$
\triangle_{i j} h_{1}^{*} \equiv h_{1}\left(y_{2}^{i}, x, r_{1}^{*}\right)-h_{1}\left(y_{2}^{j}, x, r_{1}^{*}\right)
$$

Here $y_{2}^{i}$ and $y_{2}^{j}$ are two interior points of support of the distribution of $Y_{2}$ with, $r_{1}^{*}$ is a value of $\rho_{1}$ defined as

$$
r_{1}^{*} \equiv Q_{\rho_{1} \mid \rho_{2} X}\left(\tau_{1} \mid \tau_{2}, x\right)
$$

where $\tau \equiv\left\{\tau_{1}, \tau_{2}\right\} \in(0,1)^{2}$ and $r_{1}^{*}$ is invariant with respect to choice of $x \in \tilde{x}_{i j}$, a set of instrumental values of $X$.

The identification strategy involves putting in place conditions sufficient to identify the values of the two structural functions that appear in the partial difference. Then additional restrictions ( $\mathrm{C} 1$ and $\mathrm{C} 2$ below) are introduced which make these values invariant with respect to the variations in $x$ required to identify the two values of the structural functions that appear in $\triangle_{i j} h_{1}^{*}$.

For $k \in\{i, j\}$, let $\tilde{x}_{k}$ be a set of instrumental values for which conditions B1 B4 hold, conditions sufficient to interval identify $h_{1}\left(y_{2}^{k}, x, r_{1}^{*}\right)$ with $x \in \tilde{x}_{k}$. Define $\tilde{x}_{i j} \equiv \tilde{x}_{i} \cup \tilde{x}_{j}$ and consider conditions $\mathrm{C} 1-\mathrm{C} 2$.

(C1) The value of $r_{1}^{*}$ is invariant with respect to choice of $x \in \tilde{x}_{i j}$.

(C2) For $k \in\{i, j\}$ and $\left\{x^{\prime}, x^{\prime \prime}\right\} \in \tilde{x}_{i j}$ :

$$
h_{1}\left(y_{2}^{k}, x^{\prime}, r_{1}^{*}\right)=h_{1}\left(y_{2}^{k}, x^{\prime \prime}, r_{1}^{*}\right) .
$$

Under these conditions there are the interval identifying inequalities:

$$
\begin{aligned}
q^{L}\left(\tau, y_{2}^{i}, \tilde{x}_{i}\right) & \leq h_{1}\left(y_{2}^{i}, x, r_{1}^{*}\right) \leq q^{U}\left(\tau, y_{2}^{i}, \tilde{x}_{i}\right) \\
-q^{U}\left(\tau, y_{2}^{j}, \tilde{x}_{j}\right) & \leq-h_{1}\left(y_{2}^{j}, x, r_{1}^{*}\right) \leq-q^{L}\left(\tau, y_{2}^{j}, \tilde{x}_{j}\right)
\end{aligned}
$$

where $x \in \tilde{x}_{i j}$. Addition yields an inequality which interval identifies the structural partial difference $\triangle_{i j} h_{1}^{*}$, a result formally expressed in Theorem 4 .

\footnotetext{
${ }^{16}$ This local point identification result is given in Chesher (2003).
} 
Theorem 4. Suppose that (i) Restriction A1 holds, (ii) with $\{i, j\} \subseteq\{2, \ldots, M-$ $1\}$, for $k \in\{i, j\}, \tilde{x}_{k} \equiv\left\{x^{k-1}, x^{k}\right\}$ is such that the conditions of Theorem 1 hold when identification of the value of $h_{1}\left(y_{2}^{k}, x, r_{1}^{*}\right)$ is considered and $x \in \tilde{x}_{k}$, (iii) restrictions C1 and C2 hold. Then:

$$
q^{L}\left(\tau, y_{2}^{i}, \tilde{x}_{i}\right)-q^{U}\left(\tau, y_{2}^{j}, \tilde{x}_{j}\right) \leq \triangle_{i j} h_{1}^{*} \leq q^{U}\left(\tau, y_{2}^{i}, \tilde{x}_{i}\right)-q^{L}\left(\tau, y_{2}^{j}, \tilde{x}_{j}\right)
$$

where

$$
\triangle_{i j} h_{1}^{*} \equiv h_{1}\left(y_{2}^{i}, x, r_{1}^{*}\right)-h_{1}\left(y_{2}^{j}, x, r_{1}^{*}\right)
$$

and $x \in \tilde{x}_{i j} \equiv \tilde{x}_{i} \cup \tilde{x}_{j}$.

The proof is omitted, being straightforward following the argument outlined above.

3.2. Identification of a partial difference with respect to covariates. Partition $x$ thus $x=[\hat{x}, \check{x}]$ and write the structural function thus: $h_{1}\left(y_{2}, \hat{x}, \check{x}, \rho_{1}\right)$ making explicit the dependence on the components of $x$. This Section provides conditions under which there is identification of the value of the structural partial difference $\triangle_{a b} h_{1}^{*}$ defined as follows.

$$
\triangle_{a b} h_{1}^{*} \equiv h_{1}\left(y_{2}^{i}, \hat{x}_{a}, \check{x}, r_{1}^{*}\right)-h_{1}\left(y_{2}^{i}, \hat{x}_{b}, \check{x}, r_{1}^{*}\right)
$$

Here $y_{2}^{i}$ is a point of support of the distribution of $Y_{2}$ with $i \in\{2, \ldots, M-1\}, r_{1}^{*}$ is a value of $\rho_{1}$ defined as

$$
r_{1}^{*} \equiv Q_{\rho_{1} \mid \rho_{2} X}\left(\tau_{1} \mid \tau_{2}, x\right)
$$

where $\tau \equiv\left\{\tau_{1}, \tau_{2}\right\} \in(0,1)^{2}$ and $r_{1}^{*}$ is invariant with respect to choice of $x \in \tilde{x}_{a b}$, a set of instrumental values of $X$.

For $c \in\{a, b\}$ let $\tilde{x}_{c}$ be a set of instrumental values for which conditions B1 - B4 hold, conditions sufficient to interval identify $h_{1}\left(y_{2}^{i}, \hat{x}_{c}, \check{x}, r_{1}^{*}\right)$ with $x \in \tilde{x}_{c} \equiv\left\{x_{c}^{i-1}, x_{c}^{i}\right\}$ where

$$
\begin{aligned}
x_{c}^{i-1} & \equiv\left[\hat{x}_{c}, \check{x}_{c}^{i-1}\right] \\
x_{c}^{i} & \equiv\left[\hat{x}_{c}, \check{x}_{c}^{i}\right]
\end{aligned}
$$

so that the component $\hat{x}$ takes a common value, $\hat{x}_{c}$, in $x_{c}^{i-1}$ and $x_{c}^{i}$. Define $\tilde{x}_{a b} \equiv \tilde{x}_{a} \cup \tilde{x}_{b}$ and consider conditions D1 and D2.

(D1) The value of $r_{1}^{*}$ is invariant with respect to choice of $x \in \tilde{x}_{a b}$.

(D2) For $c \in\{a, b\}$ and $\left\{\check{x}^{\prime}, \check{x}^{\prime \prime}\right\} \in\left\{\check{x}_{a}^{i-1}, \check{x}_{a}^{i}, \check{x}_{b}^{i-1}, \check{x}_{b}^{i}\right\}$ :

$$
h_{1}\left(y_{2}^{i}, \hat{x}_{c}, \check{x}^{\prime}, r_{1}^{*}\right)=h_{1}\left(y_{2}^{i}, \hat{x}_{c}, \check{x}^{\prime \prime}, r_{1}^{*}\right) .
$$


Condition D2 is clearly satisfied if $\check{x}$ is excluded from $h_{1}$. Under these conditions there are the interval identifying inequalities:

$$
\begin{aligned}
q^{L}\left(\tau, y_{2}^{i}, \tilde{x}_{a}\right) & \leq h_{1}\left(y_{2}^{i}, \hat{x}_{a}, \check{x}, r_{1}^{*}\right) \leq q^{U}\left(\tau, y_{2}^{i}, \tilde{x}_{a}\right) \\
-q^{U}\left(\tau, y_{2}^{i}, \tilde{x}_{b}\right) & \leq-h_{1}\left(y_{2}^{i}, \hat{x}_{b}, \check{x}, r_{1}^{*}\right) \leq-q^{L}\left(\tau, y_{2}^{i}, \tilde{x}_{b}\right)
\end{aligned}
$$

where $x \in \tilde{x}_{a b}$. Addition yields an inequality which interval identifies the structural partial difference $\triangle_{a b} h_{1}^{*}$, a result formally expressed in the following Theorem.

Theorem 5. Suppose that (i) Restriction A1 holds, (ii) with $i \in\{2, \ldots, M-1\}$, for $c \in\{a, b\}, \tilde{x}_{c} \equiv\left\{x_{c}^{i-1}, x_{c}^{i}\right\}$ with

$$
\begin{aligned}
x_{c}^{i-1} & \equiv\left[\hat{x}_{c}, \check{x}_{c}^{i-1}\right] \\
x_{c}^{i} & \equiv\left[\hat{x}_{c}, \check{x}_{c}^{i}\right]
\end{aligned}
$$

is such that the conditions of Theorem 1 hold when identification of the value of $h_{1}\left(y_{2}^{i}, \hat{x}_{c}, \check{x}_{c}, r_{1}^{*}\right)$ is considered, (iii) restrictions D1 and D2 hold. Then :

$$
q^{L}\left(\tau, y_{2}^{i}, \tilde{x}_{a}\right)-q^{U}\left(\tau, y_{2}^{i}, \tilde{x}_{b}\right) \leq \triangle_{a b} h_{1}^{*} \leq q^{U}\left(\tau, y_{2}^{i}, \tilde{x}_{a}\right)-q^{L}\left(\tau, y_{2}^{i}, \tilde{x}_{b}\right)
$$

where

$$
\triangle_{a b} h_{1}^{*} \equiv h_{1}\left(y_{2}^{i}, \hat{x}_{a}, \check{x}, r_{1}^{*}\right)-h_{1}\left(y_{2}^{i}, \hat{x}_{b}, \check{x}, r_{1}^{*}\right)
$$

and $x \in \tilde{x}_{a b} \equiv \tilde{x}_{a} \cup \tilde{x}_{b}$ and $\check{x} \in\left\{\check{x}_{a}^{i-1}, \check{x}_{a}^{i}, \check{x}_{b}^{i-1}, \check{x}_{b}^{i}\right\}$.

The proof is omitted, being straightforward following the argument outlined above. If an element of $X$ exhibits continuous variation and $h_{1}$ is differentiable with respect to that element then a simple extension of Theorem 5 leads to a result on interval identification of a partial derivative of $h_{1}$ with respect to a covariate.

3.3. Minimum length identifying intervals. Consider the length of an interval that identifies a partial difference, for example with respect to a discrete endogenous variable.

$$
L_{i j} \equiv\left(q^{U}\left(\tau, y_{2}^{i}, \tilde{x}_{i}\right)-q^{L}\left(\tau, y_{2}^{j}, \tilde{x}_{j}\right)\right)-\left(q^{L}\left(\tau, y_{2}^{i}, \tilde{x}_{i}\right)-q^{U}\left(\tau, y_{2}^{j}, \tilde{x}_{j}\right)\right)
$$

This is necessarily nonnegative and can be written as the sum of the lengths of the identifying intervals for the two components of the difference, also nonnegative, as follows.

$$
L_{i j}=\left(q^{U}\left(\tau, y_{2}^{i}, \tilde{x}_{i}\right)-q^{L}\left(\tau, y_{2}^{i}, \tilde{x}_{i}\right)\right)+\left(q^{U}\left(\tau, y_{2}^{j}, \tilde{x}_{j}\right)-q^{L}\left(\tau, y_{2}^{j}, \tilde{x}_{j}\right)\right)
$$

Therefore choices of $\tilde{x}_{i}$ and $\tilde{x}_{j}$ which minimise the lengths of the identifying intervals for respectively $h_{1}\left(y_{2}^{i}, x, r_{1}^{*}\right)$ and $h_{1}\left(y_{2}^{j}, x, r_{1}^{*}\right)$ yield minimum length identifying intervals for the partial difference $\triangle_{i j} h_{1}^{*}$. So an estimate of a minimum length identifying interval for a structural partial difference can be obtained by combining estimates of minimum length intervals for each component of the difference.

Point identification of a structural partial difference cannot be achieved when $Y_{1}$ is continuous and $Y_{2}$ is discrete under the weak nonparametric restrictions considered here because, under those conditions, point identification of values delivered by structural functions is infeasible. 


\section{Weak instruments}

Crucial to the identification of the value of a structural function at a point of support, $y_{2}^{m}$, of a discrete endogenous variable is the ability to find instrumental values, $x^{m-1}$ and $x^{m}$ such that for a chosen value of $\tau_{2} \in(0,1)$ the following inequality of Restriction B1 of Theorem 1 is satisfied.

$$
P\left[Y_{2} \leq y_{2}^{m} \mid X=x^{m}\right] \equiv p^{m}\left(x^{m}\right) \leq \tau_{2} \leq p^{m-1}\left(x^{m-1}\right) \equiv P\left[Y_{2} \leq y_{2}^{m-1} \mid X=x^{m-1}\right]
$$

When $X$ has only a weak effect on the conditional distribution of $Y_{2}$ given $X$ or when the support of $X$ is limited this may not be possible. The model and data studied by Angrist and Krueger (1991) (AK) illustrate the difficulty.

AK consider a parametric model in which the log wage, $Y_{1}$, is a linear function of endogenous years of schooling, $Y_{2}$. The returns to schooling is the focus of interest, that is the coefficient on $Y_{2}$ in the linear structural log wage equation.

AK propose binary indicators of quarter of birth (QOB) as instrumental variables. They argue that QOB is an influence on years of schooling because people born in different quarters of the year enter school at different ages, yet can leave school at any point in the year. The influence of QOB on attained years of schooling is evident in the data, but the effect is rather weak. AK argue that QOB does not influence the wage given years of schooling and assume that the latent variable in the structural wage equation is mean independent of QOB. Their parametric model, which incorporates these restrictions, identifies the returns to schooling.

In this Section the identifying power of the QOB instruments in the absence of AK's parametric restrictions is considered.

4.1. Discrete years of schooling. The AK data record years of schooling as integers, so $Y_{2}$ is discrete. The instrumental variables employed by AK are binary variables, $X \equiv\left[X_{1}, \ldots, X_{4}\right]$ indicating quarter of birth with $X_{i}=1$ for a person born in quarter $i$ and equal to zero otherwise. These are weak instruments so far as Restriction B1 is concerned because variation in their values can only exert a small influence on the distribution of years of schooling given $X$. For someone who leaves school at the earliest legal opportunity, say at age 15, the difference in schooling comparing people born in quarter 1 and quarter 4 is only a few months.

Table 1 shows nonparametric estimates of the distribution function of years of schooling for each quarter of birth. ${ }^{17}$ The data come from 329, 509 members of the 1930-39 cohort observed in 1980, around 80,000 born in each of the four quarters. ${ }^{18}$ The QOB 1 and QOB 4 estimated distribution functions are plotted in Figure 1.

\footnotetext{
${ }^{17}$ These are the empirical distribution functions. The estimate of $P\left[Y_{2} \leq y_{2}^{m} \mid X_{i}=1\right]$ is simply the proportion of those with $X_{i}=1$ having recorded years of schooling no greater than $y^{m}$.

${ }^{18}$ Sample sizes used in producing each entry in a row of Table 1 exceed 1,000 for $Y_{2}>4$ and exceed 7,000 for $Y_{2}>7$ after which the estimates are very accurate. For example the estimated standard errors at $Y_{2}=8$ are all close to 0.0033 .
} 
Table 1: Estimated distribution function of years of schooling for each quarter of birth

\begin{tabular}{|l|l|l|l|l|}
\hline $\begin{array}{c}\text { Years of } \\
\text { schooling }\end{array}$ & $\begin{array}{c}\text { QOB } \\
1\end{array}$ & $\begin{array}{c}\text { QOB } \\
2\end{array}$ & $\begin{array}{c}\text { QOB } \\
3\end{array}$ & $\begin{array}{c}\text { QOB } \\
4\end{array}$ \\
\hline 1 & .002 & .003 & .002 & .002 \\
\hline 2 & .005 & .005 & .004 & .004 \\
\hline 3 & .008 & .009 & .007 & .007 \\
\hline 4 & .013 & .013 & .011 & .010 \\
\hline 5 & .020 & .019 & .016 & .016 \\
\hline 6 & .032 & .032 & .028 & .027 \\
\hline 7 & .052 & .050 & .046 & .045 \\
\hline 8 & .104 & .101 & .094 & .090 \\
\hline 9 & .146 & .142 & .133 & .128 \\
\hline 10 & .197 & .194 & .182 & .177 \\
\hline 11 & .238 & .236 & .223 & .218 \\
\hline 12 & .612 & .604 & .598 & .595 \\
\hline 13 & .666 & .658 & .653 & .652 \\
\hline 14 & .742 & .734 & .734 & .732 \\
\hline 15 & .771 & .763 & .764 & .763 \\
\hline 16 & .877 & .873 & .874 & .872 \\
\hline 17 & .913 & .909 & .911 & .909 \\
\hline 18 & .947 & .945 & .947 & .946 \\
\hline 19 & .966 & .964 & .966 & .966 \\
\hline 20 & 1.00 & 1.00 & 1.00 & 1.00 \\
\hline
\end{tabular}

Inspecting Table 1 it can be seen that for every value of $m$

$$
\max _{i}\left(\hat{P}\left[Y_{2} \leq y_{2}^{m-1} \mid X_{i}=1\right]\right)<\min _{i}\left(\hat{P}\left[Y_{2} \leq y_{2}^{m-1} \mid X_{i}=1\right]\right)
$$

and it follows that there is no value of $\tau_{2}$ and $m$ for which there are configurations of the four binary indicators, $x^{m}$ and $x^{m-1}$ such that:

$$
\hat{p}^{m}\left(x^{m}\right) \leq \tau_{2} \leq \hat{p}^{m-1}\left(x^{m-1}\right) .
$$

This strongly suggests that the AK quarter of birth instruments are too weak to nonparametrically identify the returns to schooling.

4.2. "Continuous" years of schooling. It is interesting to consider what the identifying power of the quarter of birth instruments would have been had years of schooling been recorded continuously. This also provides the opportunity to illustrate some of the results set out earlier in the paper. 
For this exercise the estimated distribution functions reported in Table 1 are treated as if they were the actual distribution functions. The integer years of schooling recorded in the AK data are treated as if they were the result of rounding exact figures to the nearest integer in a situation in which a value recorded as $y_{2}$ is equally likely to be any value in the interval $y_{2} \pm 0.5$. Figure 2 shows the resulting hypothetical distribution functions of continuous years of schooling for QOB 1 (solid) and QOB 4 (dashed). ${ }^{19}$

Write the structural log wage function as

$$
Y_{1}=h_{1}\left(Y_{2}, X, \rho_{1}\right)
$$

where $Y_{1}$ denotes the log wage, $X \equiv\left\{X_{1}, X_{2}, X_{3}, X_{4}\right\}$ lists the QOB indicators and $Y_{2}$ denotes schooling. Let $x_{[i]}$ denote the configuration of QOB indicators in which $X_{i}=1$.

Section 2.8 considered nonparametric identification in the presence of a continuous endogenous variable. Suppose that, for some $\tau_{2}$ and $y_{2}^{*}$, there are QOB configurations $x_{[i]}$ and $x_{[j]},\{i, j\} \subset\{1,2,3,4\}$, not necessarily distinct, such that the inequality

$$
F_{Y_{2} \mid X}\left(y_{2}^{*} \mid x_{[i]}\right) \leq \tau_{2} \leq F_{Y_{2} \mid X}\left(y_{2}^{*} \mid x_{[j]}\right)
$$

is satisfied. The discussion of the remainder of this Section is predicated on the validity of the other required identifying restrictions. ${ }^{20}$

If (19) holds there is interval identification of the value of the log wage structural function evaluated at $Y_{2}=y_{2}^{*}, X=x \in\left\{x_{[i]}, x_{[j]}\right\}$ and

$$
\rho_{1}=r_{1}^{*}\left(\tau_{2}\right) \equiv Q_{\rho_{1} \mid \rho_{2} X}\left(\tau_{1} \mid \tau_{2}, x\right) .
$$

It is convenient to make the dependence of $r_{1}^{*}$ on $\tau_{2}$ explicit here. The additional identifying restrictions ensure that $h_{1}$ and $r_{1}^{*}\left(\tau_{2}\right)$ are invariant to choice of $x \in\left\{x_{[i]}, x_{[j]}\right\}$.

Figure 3 zooms in on Figure 2, focussing on years of schooling between 5 and 12 years. The vertical line in Figure 3 marks 8 years of schooling. It intersects the distribution functions at $a=.109$ and at $b=.125$. Considering the inequality (19) there is therefore interval identification of $h_{1}\left(8, x, r_{1}^{*}\left(\tau_{2}\right)\right)$ for $x \in\left\{x_{[1]}, x_{[4]}\right\}$ at all values $\tau_{2} \in[.109, .125]$, and for $\tau_{2}$ in this interval:

$$
\begin{gathered}
\min \left(Q_{Y_{1} \mid Y_{2} X}\left(\tau_{1} \mid 8, x_{[1]}\right), Q_{Y_{1} \mid Y_{2} X}\left(\tau_{1} \mid 8, x_{[4]}\right)\right) \\
\leq h_{1}\left(8, x, r_{1}^{*}\left(\tau_{2}\right)\right) \leq \\
\max \left(Q_{Y_{1} \mid Y_{2} X}\left(\tau_{1} \mid 8, x_{[1]}\right), Q_{Y_{1} \mid Y_{2} X}\left(\tau_{1} \mid 8, x_{[4]}\right)\right)
\end{gathered}
$$

\footnotetext{
${ }^{19}$ These conjectured distribution functions are obtained by joining midpoints of each successive step of each of the estimated distribution functions graphed in Figure 1. The midpoints are joined with straight lines because the result is intended to reflect the distribution of "continuous" years of schooling in the situation in which schooling is uniformly distributed within each one year interval. Alternative "assumptions" about the distribution of schooling within one year intervals are captured using alternative increasing functions to join the midpoints.

${ }^{20}$ Thus it is supposed that $h_{1}$ and $Q_{\rho_{1} \mid \rho_{2} X}\left(\tau_{1} \mid \tau_{2}, x\right)$ are insensitive to variations in $x \in\left\{x_{[i]}, x_{[j]}\right\}$ and the required montonicity conditions hold.
} 
where $x \in\left\{x_{[1]}, x_{[4]}\right\}$.

At $\tau_{2}=.109$ and $\tau_{2}=.125$ the structural $\log$ wage function $h_{1}\left(8, x, r_{1}^{*}\left(\tau_{2}\right)\right)$ is point identified because in (19)

$$
\begin{aligned}
& F_{Y_{2} \mid X}\left(8 \mid x_{[1]}\right) \leq .109 \leq F_{Y_{2} \mid X}\left(8 \mid x_{[1]}\right) \\
& F_{Y_{2} \mid X}\left(8 \mid x_{[4]}\right) \leq .125 \leq F_{Y_{2} \mid X}\left(8 \mid x_{[4]}\right)
\end{aligned}
$$

which are of course equalities, and so:

$$
\begin{aligned}
& h_{1}\left(8, x, r_{1}^{*}(.109)\right)=Q_{Y_{1} \mid Y_{2} X}\left(\tau_{1} \mid 8, x_{[1]}\right) \\
& h_{1}\left(8, x, r_{1}^{*}(.125)\right)=Q_{Y_{1} \mid Y_{2} X}\left(\tau_{1} \mid 8, x_{[4]}\right)
\end{aligned}
$$

where $x \in\left\{x_{[1]}, x_{[4]}\right\}$.

Now consider the horizontal line drawn in Figure 3 at $\tau_{2}=0.2$. This intersects the distribution functions at $c=9.58$ and $d=10.06$. Considering the inequality (19) there is

$$
\begin{aligned}
F_{Y_{2} \mid X}\left(9.58 \mid x_{[1]}\right) & \leq 0.2 \leq F_{Y_{2} \mid X}\left(9.58 \mid x_{[1]}\right) \\
F_{Y_{2} \mid X}\left(10.06 \mid x_{[4]}\right) & \leq 0.2 \leq F_{Y_{2} \mid X}\left(10.06 \mid x_{[4]}\right)
\end{aligned}
$$

and therefore at $y_{2}=9.58$ and $y_{2}=10.06$ the value of the structural function $h_{1}\left(y_{2}, x, r_{1}^{*}(0.2)\right)$ is point identified, thus:

$$
\begin{aligned}
h_{1}\left(9.58, x, r_{1}^{*}(0.2)\right) & =Q_{Y_{1} \mid Y_{2} X}\left(\tau_{1} \mid 9.58, x_{[1]}\right) \\
h_{1}\left(10.06, x, r_{1}^{*}(0.2)\right) & =Q_{Y_{1} \mid Y_{2} X}\left(\tau_{1} \mid 10.06, x_{[4]}\right)
\end{aligned}
$$

where $x \in\left\{x_{[1]}, x_{[4]}\right\}$.

For any choice of $\tau_{1}$ the structural functions in (22) and (23) are evaluated at the same value of $\rho_{1}$, that is

$$
r_{1}^{*}(0.2) \equiv Q_{\rho_{1} \mid \rho_{2} X}\left(\tau_{1} \mid 0.2, x\right) .
$$

Therefore the difference between the quantile functions in (22) and (23) point identifies a partial difference of the structural function with respect to years of schooling. The returns to schooling to increasing schooling by 0.48 years, from 9.58 years to 10.06 years, for a person at the 0.2 -quantile of the distribution of $\rho_{2}$ and the $\tau_{1}$-quantile of the distribution of $\rho_{1}$ conditional on $\tau_{2}=0.2$ and $x \in\left\{x_{[1]}, x_{[4]}\right\}$ is identified by

$$
\frac{1}{0.48}\left(Q_{Y_{1} \mid Y_{2} X}\left(\tau_{1} \mid 10.06, x_{[4]}\right)-Q_{Y_{1} \mid Y_{2} X}\left(\tau_{1} \mid 9.58, x_{[1]}\right)\right) \text {. }
$$

Returning to Figure 3, moving the horizontal line away from $\tau_{2}=0.2$ produces a sequence of pairs of values of years of schooling generated by the intersection of the horizontal line with the two estimated distribution functions. These are the pairs of values at which the returns to schooling can be identified. 
The binary and weak nature of the QOB instruments have significant implications in the absence of parametric restrictions. Because the two QOB instruments are binary each pair of values is associated with a different value of $\tau_{2}$. One could never learn about the returns to schooling at low levels of schooling for a person at a high quantile of the distribution of $\rho_{2}$. Because the instruments are weak the pairs of values exhibit very little separation. One could not learn about the returns to a large change in schooling.

\section{Concluding Remarks}

5.1. Local identification. The objects whose identification is studied in this paper are local features of structures: values and partial differences of structural functions at specific values of their arguments. The conditions under which they are identified are specific to the objects considered and local in character: for example they require structural functions to be locally insensitive to particular variations in covariates; they require insensitivity of a specific quantile of a latent variate to particular variations in covariates.

Local conditions of this sort allow one to understand when a structural interpretation can be placed on particular features of estimated conditional quantile and distribution functions. Nonparametric identification of entire nonseparable structural functions seems to require the unpalatable restriction that latent variates and covariates are independently distributed as in the analysis of Roehrig (1988), Imbens and Newey (2003) and Matzkin (2003). One might be prepared to believe in a degree of local independence even in situations in which global independence seems untenable.

5.2. Many endogenous variables. The results given here are for the case in which there is a single endogenous variable. Extension to the case in which one endogenous variable $\left(Y_{2}\right)$ is discrete and there is another continuous endogenous variable $\left(Y_{3}\right)$ is straightforward. Among the conditions under which there is interval identification of the value of a structural function at $Y_{2}=y_{2}^{m}$ and $Y_{3}=y_{3}$ is an extension of Restriction B1 in which in the inequality (11) probabilities are conditional on $X$ and on $Y_{3}=y_{3}$. Further, the instrumental values must be chosen so that $y_{3}$ is a quantile of the distribution of $Y_{3}$ given $X$ at all of the instrumental values. This may be difficult to achieve is $X$ does not have rich support but arguments like those employed here still yield interval identification when $y_{3}$ cannot be set to a chosen quantile of $Y_{3}$ by choice of $X$. When $Y_{3}$ is discrete the extension of the results given here requires extension of Lemma 2 to provide bounds on $F_{Y_{1} \mid Y_{2} Y_{3} X}\left(y_{1} \mid y_{2}^{m}, y_{3}^{n}, x\right)$ and thus bounds on conditional quantiles of $Y_{1}$.

5.3. Binary endogenous variables. The conditions employed in this paper exploit the ordering of discrete outcomes but when an endogenous variable is binary there is no ordering of outcomes. Nonparametric identification of features of nonseparable structural functions that depend on binary endogenous variables requires stronger conditions than those developed here. 
5.4. Classical identification analysis. The classical analysis of the identification problem encapsulated in Koopmans, Rubin and Leipnik (1950) was set in the context of a linear simultaneous equations system. That analysis produced the well known rank condition which, under the restriction that latent variates are mean independent of covariates (instruments), is necessary and sufficient for the identification of the values of the coefficients of a linear structural function of the system.

This rank condition requires that there are covariates, variation in which has effect on the conditional means of the endogenous variables that appear in the structural function and has no direct effect on the structural functions.

The identification conditions deployed in this paper are extensions of these classical conditions, constructed within the framework for the study of parametric and nonparametric identification proposed by Hurwicz (1950). ${ }^{21}$ The mean independence covariation restriction of Koopmans, Rubin and Leipnik (1950) must be modified when a structural function is nonseparable in its latent variate because, when a structural functions is nonseparable in its latent variate, conditional mean independence is an uninformative restriction unless there are highly restrictive conditions on the functional form of the structural function. If the structural function is restricted to be monotonic in its latent variate, a restriction of course satisfied in a separable model, a conditional quantile restriction is informative when a structural function is nonseparable.

The rank condition of the classical analysis appears here in the requirement that (a) there exist variation in covariates with an effect on the conditional distribution function of an endogenous variate given covariates of sufficient magnitude, as set out in Restriction B1, and (b) that the structural function is insensitive to this variation.

\footnotetext{
${ }^{21}$ This early development by Leonid Hurwicz is remarkable given that it was done at a time when linear separable parametric models preoccupied most people working in econometrics. The contribution is clearly acknowledged in Koopmans and Reiersøl (1950): "We shall therefore use the terms and concepts introduced by Hurwicz ... which cover both parametric and non-parametric specifications". Hurwicz (1950) is one of five papers which Koopmans "draws freely on" in his expository paper Koopmans (1949), the others authored by Koopmans and Rubin, Wald, Koopmans and Reiersøl, and Leonid Hurwicz himself.
} 


\section{REFERENCES}

Angrist, Joshua, D., And Alan B. Krueger (1991): "Does compulsory schooling attendance affect schooling and earnings?" Quarterly Journal of Economics, 106, 979 - 1014.

Becker, Gary S., And Barry R. Chiswick (1966): "Education and the distribution of earnings," American Economic Review, 56, 358-369.

Bound, John, David A. Jaeger and Regina M. Baker (1995): "Problems with Instrumental Variables Estimation when the Correlation Between the Instruments and the Endogenous Explanatory Variable is Weak," Journal of the American Statistical Association, 90, 443 - 450.

CARD, David (2001): "Estimating the returns to schooling: Progress on some persistent econometric problems," Econometrica, 69, 1127-1160.

Chaudhuri, Probal (1991): "Nonparametric estimation of regression quantiles and their local Bahadur representation," Annals of Statistics, 19, 760-777.

Chaudhuri, Probal, Kjell Doksum and Alexander Samarov (1997): "On average derivative quantile regression," Annals of Statistics, 25, 715-744.

Chernozhukov, Victor, Han Hong and Elie Tamer (2003): "Parameter Set Inference in a class of Econometric Models," manuscript.

Chesher, Andrew D. (2002): "Instrumental values," Centre for Microdata Methods and Practice Working Paper 17/02.

Chesher, Andrew D. (2003): "Identification in nonseparable models," Econometrica, 71, 1404 - 1441.

CHISWICK, BARRY R. (1974): Income inequality: regional analyses within a human capital framework. New York: Columbia University Press.

Chiswick, Barry R., And Jacob Mincer (1972): "Time series changes in personal income inequality," Journal of Political Economy, 80, S34-S66.

Cockburn, Iain, And Zvi Griliches (1988): "The Estimation and Measurement of Spillover Effects of R\&D Investment," American Economic Review, 78, No. 2, Papers and Proceedings of the One-Hundredth Annual Meeting of the American Economic Association, 419 - 423.

Darolles, Serge, Jean-Pierre Florens and Eric Renault, (2000): "Nonparametric instrumental regression," CREST Documents de Travail 2000-17.

Das, Mitali (2003): "Instrumental Variables Estimation of Nonparametric Models with Discrete Endogenous Variables," forthcoming, Journal of Econometrics.

Dubin, Jeffrey, A., Daniel. L. McFadden (1984): "An Econometric Analysis of Residential Electrical Appliance Holdings and Consumption," Econometrica, 52, $345-362$.

Florens, Jean-Pierre and Laetitia Malavolti (2003): "Instrumental Regression with Discrete Variables," presented at the 2003 European Meeting of the Econometric Society, Stockholm.

Griliches, Zvi (1990): "Patent Statistics as Economic Indicators: A Survey," Journal of Economic Literature, 28, 1661 - 1707. 
Hurwicz, LeOnid (1950): "Generalization of the concept of identification," in Statistical inference in dynamic economic models. Cowles Commission Monograph 10, New York, John Wiley.

Imbens, Guido W., And Whitney K. Newey (2003): "Identification and estimation of triangular simultaneous equations models without additivity," Manuscript, paper presented at the 14 th $\mathrm{EC}^{2}$ Meeting, cemmap, London, December 12th - 13th 2003.

Imbens, Guido W., And Charles F. Manski (2003): "Confidence Intervals for Partially Identified Parameters," Centre for Microdata Methods and Practice Working Paper 09/03.

Kahn, Shakeeb (2001): "Two-stage rank estimation of quantile index models," Journal of Econometrics, 100, 319-355.

Koenker, Roger W., And Gilbert W. Bassett JR. (1978): "Regression quantiles," Econometrica, 46, 33-50.

Koenker, Roger W. And Vasco D'Orey (1987): "Computing regression quantiles," Journal of the Royal Statistical Society, Series C, 36, 383-393.

Koopmans, TJalling C., (1949): "Identification Problems in Economic Model Construction," Econometrica, 17, 125 - 144.

Koopmans, Tualling C., and Olav Reiersøl (1950): "The identification of structural characteristics," Annals of Mathematical Statistics, 21, 165-181.

Koopmans, Tualling C., Herman Rubin and Roy B. Leipnik (1950): "Measuring the Equation Systems of Dynamic Economics," in Statistical inference in dynamic economic models. Cowles Commission Monograph 10, New York, John Wiley. Lee, Sokbae (2003a): "Efficient Semiparametric Estimation of a Partially Linear Quantile Regression Model," Econometric Theory, 19, 1-31.

LeE, Sokbae (2003b): "Endogeneity in Quantile Regression Models: a Control Function Approach," Manuscript.

Ma, Linguie And Roger W. Koenker (2003): "Quantile Regression Methods for Recursive Structural Equation Models," Manuscript.

Machado, José A.F., And Joao M.C. Santos Silva (2002): "Quantiles for Counts," Centre for Microdata Methods and Practice Working Paper 22/02.

Matzkin, Rosa L. (2003): "Nonparametric Estimation of Nonadditive Random Functions," Econometrica, 71, 1339 - 1376.

MinceR, JАCOB (1974): Schooling experience and earnings. New York: Columbia University Press.

Newey, Whitney K., And James L. Powell (2003): "Instrumental Variables Estimation for Nonparametric Models," Econometrica, 71, 1565 - 1578.

Newey, Whitney K., James L. Powell, and Frank Vella (1999): "Nonparametric Estimation of Triangular Simultaneous Equations Models," Econometrica 67, 565-603.

PINKSE, JoRIS (2000): "Nonparametric two-step regression functions when regressors and errors are dependent," Canadian Journal of Statistics, 28, 289-300. 
Roehrig, Charles S. (1988): "Conditions for identification in nonparametric and parametric models", Econometrica 56, 433-447.

Severini, Thomas, and Gautam Tripathi (2003): "Some Identification Issues in Nonparametric Linear Regression with Endogenous Regressors," Manuscript.

\section{Appendix: Proofs of Theorems}

First, two lemmata employed in the proofs of the theorems are stated and proved. The inverse function $h_{1}^{-1}$ will be used, defined as follows.

$$
h_{1}^{-1}\left(y_{2}^{m}, x, y_{1}\right) \equiv \sup _{p}\left\{p: h_{1}\left(y_{2}^{m}, x, p\right) \leq y_{1}\right\}
$$

When $h_{1}\left(y_{2}, x, \rho_{1}\right)$ varies strictly monotonically with $\rho_{1}$ this is the conventional inverse function satisfying

$$
y_{1}=h_{1}\left(y_{2}^{m}, x, h_{1}^{-1}\left(y_{2}^{m}, x, y_{1}\right)\right)
$$

for all $y_{2}^{m}, x$ and $y_{1}$.

\section{Lemma 1}

Under Restriction (I) the conditional distribution and $\tau_{1}$-quantile functions of $Y_{1}$ given $Y_{2}=y_{2}^{m}$ and $X=x$ are as follows.

$$
\begin{gathered}
F_{Y_{1} \mid Y_{2} X}\left(y_{1} \mid y_{2}^{m}, x\right)=\frac{1}{p_{m}(x)} \int_{p^{m-1}(x)}^{p^{m}(x)} F_{\rho_{1} \mid \rho_{2} X}\left(h_{1}^{-1}\left(y_{2}^{m}, x, y_{1}\right) \mid r_{2}, x\right) d r_{2} \\
Q_{Y_{1} \mid Y_{2} X}\left(\tau_{1} \mid y_{2}^{m}, x\right)=\inf _{q}\left\{q: \frac{1}{p_{m}(x)} \int_{p^{m-1}(x)}^{p^{m}(x)} F_{\rho_{1} \mid \rho_{2} X}\left(h_{1}^{-1}\left(y_{2}^{m}, x, q\right) \mid r_{2}, x\right) d r_{2} \geq \tau_{1}\right\}
\end{gathered}
$$

\section{Proof of Lemma 1}

The conditional distribution function of $Y_{1}$ given $Y_{2}=y_{2}^{m}$ and $X=x$ is defined as follows.

$$
F_{Y_{1} \mid Y_{2} X}\left(y_{1} \mid y_{2}^{m}, x\right) \equiv \frac{P\left[Y_{1} \leq y_{1} \cap Y_{2}=y_{2}^{m} \mid X=x\right]}{P\left[Y_{2}=y_{2}^{m} \mid X=x\right]}
$$

Given $X=x$, in terms of events, since $h_{1}$ is a non-decreasing function of $\rho_{1}$,

$$
\left\{Y_{1} \leq y_{1} \cap Y_{2}=y_{2}^{m}\right\}=\left\{\rho_{1} \leq h_{1}^{-1}\left(y_{2}^{m}, x, y_{1}\right) \cap\left\{p^{m-1}(x)<\rho_{2} \leq p^{m}(x)\right\}\right.
$$

and so

$$
P\left[Y_{1} \leq y_{1} \cap Y_{2}=y_{2}^{m} \mid X=x\right]=\int_{p^{m-1}(x)}^{p^{m}(x)} F_{\rho_{1} \mid \rho_{2} X}\left(h_{1}^{-1}\left(y_{2}^{m}, x, y_{1}\right) \mid r_{2}, x\right) d r_{2}
$$

and the expression for $F_{Y_{1} \mid Y_{2} X}\left(y_{1} \mid y_{2}^{m}, x\right)$ is delivered on dividing by $p_{m}(x) \equiv P\left[Y_{2}=\right.$ $\left.y_{2}^{m} \mid X=x\right]$. The expression for the conditional quantile function follows directly. 
Lemma 2 places bounds on the conditional quantile function of $Y_{1}$ conditional on $Y_{2}=y_{2}^{m}$ and $X=x$.

\section{Lemma 2}

Assume Restriction A1 holds. If for all $r_{1}, F_{\rho_{1} \mid \rho_{2} X}\left(r_{1} \mid r_{2}, x\right)$ is monotonic in $r_{2}$ for $r_{2} \in\left(p^{m-1}(x), p^{m}(x)\right]$ then

$$
\begin{gathered}
\min \left\{\begin{array}{c}
h_{1}\left(y_{2}^{m}, x, Q_{\rho_{1} \mid \rho_{2} X}\left(\tau_{1} \mid p^{m-1}(x), x\right)\right) \\
h_{1}\left(y_{2}^{m}, x, Q_{\rho_{1} \mid \rho_{2} X}\left(\tau_{1} \mid p^{m}(x), x\right)\right)
\end{array}\right\} \\
\leq Q_{Y_{1} \mid Y_{2} X}\left(\tau_{1} \mid y_{2}^{m}, x\right) \leq \\
\max \left\{\begin{array}{c}
h_{1}\left(y_{2}^{m}, x, Q_{\rho_{1} \mid \rho_{2} X}\left(\tau_{1} \mid p^{m-1}(x), x\right)\right) \\
h_{1}\left(y_{2}^{m}, x, Q_{\rho_{1} \mid \rho_{2} X}\left(\tau_{1} \mid p^{m}(x), x\right)\right)
\end{array}\right\} .
\end{gathered}
$$

If $\rho_{1}$ and $\rho_{2}$ are independently distributed given $X=x$ over the interval $\left[p^{m-1}(x), p^{m}(x)\right]$ in the sense that for $r_{2}$ in this interval $\left.Q_{\rho_{1} \mid \rho_{2} X}\left(\tau_{1} \mid r_{2}, x\right)\right)=r_{1}^{*}(x)$, a constant, then:

$$
Q_{Y_{1} \mid Y_{2} X}\left(\tau_{1} \mid y_{2}^{m}, x\right)=h_{1}\left(y_{2}^{m}, x, r_{1}^{*}(x)\right) .
$$

Remark A1. The weak monotonicity condition on $F_{\rho_{1} \mid \rho_{2} X}\left(r_{1} \mid r_{2}, x\right)$ is sufficient but not necessary, a degree of bounded variation being all that is required. Specifically, the result of Lemma 2 also applies if, for all $r_{1}$ in a wide enough interval around $Q_{\rho_{1} \mid \rho_{2} X}\left(\tau_{1} \mid p^{m}(x), x\right)$, there exists $\theta \in[0,1]$ such that for all $r_{2} \in\left(p^{m-1}(x), p^{m}(x)\right]$

$$
F_{\rho_{1} \mid \rho_{2} X}\left(r_{1} \mid r_{2}, x\right)=\theta F_{\rho_{1} \mid \rho_{2} X}\left(r_{1} \mid p^{m-1}(x), x\right)+(1-\theta) F_{\rho_{1} \mid \rho_{2} X}\left(r_{1} \mid p^{m}(x), x\right) .
$$

Remark A2. If the structural function depends on both latent variates, thus: $h_{1}\left(Y_{2}, X, \rho_{1}, \rho_{2}\right)$, then the result of Lemma 2 applies if for all $t_{1}$ in a wide enough interval around $\tau_{1}$, there exists $\theta \in[0,1]$ such that for all $r_{2} \in\left(p^{m-1}(x), p^{m}(x)\right]$

$$
\begin{aligned}
h_{1}\left(y_{2}^{m}, x, Q_{\rho_{1} \mid \rho_{2} X}\left(\tau_{1} \mid r_{2}, x\right), r_{2}\right)= & \theta h_{1}\left(y_{2}^{m}, x, Q_{\rho_{1} \mid \rho_{2} X}\left(\tau_{1} \mid p^{m-1}(x), x\right), p^{m-1}(x)\right) \\
& +(1-\theta) h_{1}\left(y_{2}^{m}, x, Q_{\rho_{1} \mid \rho_{2} X}\left(\tau_{1} \mid p^{m}(x), x\right), p^{m}(x)\right)
\end{aligned}
$$

for which the monotonicity of $h_{1}\left(y_{2}^{m}, x, Q_{\rho_{1} \mid \rho_{2} X}\left(\tau_{1} \mid r_{2}, x\right), r_{2}\right)$ with respect to $r_{2}$ is sufficient.

Remark A3. The bounds of Lemma 2 apply when $m=M$ but note that in that case $p^{M}(x)=1$ for all $x$. They also apply when $m=1$ with $p^{0}(x) \equiv 0$.

\section{Proof of Lemma 2}

First consider the case in which $F_{\rho_{1} \mid \rho_{2} X}$ is non-decreasing in $\rho_{2}$ for $\rho_{2} \in\left(p^{m-1}(x), p^{m}(x)\right]$. Recalling the result of Lemma 1 , replacing $\rho_{2}$ in the integrand first by $p^{m-1}(x)$ and then by $p^{m}(x)$ yields the following inequality.

$$
\frac{1}{p_{m}(x)} \int_{p^{m-1}(x)}^{p^{m}(x)} F_{\rho_{1} \mid \rho_{2} X}\left(h_{1}^{-1}\left(y_{2}^{m}, x, y_{1}\right) \mid p^{m-1}(x), x\right) d \rho_{2}
$$




$$
\begin{gathered}
\leq F_{Y_{1} \mid Y_{2} X}\left(y_{1} \mid y_{2}^{m}, x\right) \leq \\
\frac{1}{p_{m}(x)} \int_{p^{m-1}(x)}^{p^{m}(x)} F_{\rho_{1} \mid \rho_{2} X}\left(h_{1}^{-1}\left(y_{2}^{m}, x, y_{1}\right) \mid p^{m}(x), x\right) d \rho_{2}
\end{gathered}
$$

Since, for example,

$$
\begin{array}{r}
\frac{1}{p_{m}(x)} \int_{p^{m-1}(x)}^{p^{m}(x)} F_{\rho_{1} \mid \rho_{2} X}\left(h_{1}^{-1}\left(y_{2}^{m}, x, y_{1}\right) \mid p^{m}(x), x\right) d \rho_{2} \\
=F_{\rho_{1} \mid \rho_{2} X}\left(h_{1}^{-1}\left(y_{2}^{m}, x, y_{1}\right) \mid p^{m}(x), x\right)
\end{array}
$$

there is, for all $y_{1}$, the following inequality.

$$
\begin{gathered}
F_{\rho_{1} \mid \rho_{2} X}\left(h_{1}^{-1}\left(y_{2}^{m}, x, y_{1}\right) \mid p^{m-1}(x), x\right) \\
\leq F_{Y_{1} \mid Y_{2} X}\left(y_{1} \mid y_{2}^{m}, x\right) \leq \\
F_{\rho_{1} \mid \rho_{2} X}\left(h_{1}^{-1}\left(y_{2}^{m}, x, y_{1}\right) \mid p^{m}(x), x\right)
\end{gathered}
$$

When $F_{\rho_{1} \mid \rho_{2} X}$ is non-increasing in $\rho_{2}$ for $\rho_{2} \in\left(p^{m-1}(x), p^{m}(x)\right]$ the inequality (L2.1) is reversed.

Since the functions on the left and the right of the inequality (L2.1) bound $F_{Y_{1} \mid Y_{2} X}\left(y_{1} \mid y_{2}^{m}, x\right)$ respectively below and above, the values, $q_{m-1}$ and $q_{m}$ defined by:

$$
\begin{aligned}
q_{m-1} & \equiv \inf _{q}\left\{q: F_{\rho_{1} \mid \rho_{2} X}\left(h_{1}^{-1}\left(y_{2}^{m}, x, q\right) \mid p^{m-1}(x), x\right) \geq \tau_{1}\right\} \\
q_{m} & \equiv \inf _{q}\left\{q: F_{\rho_{1} \mid \rho_{2} X}\left(h_{1}^{-1}\left(y_{2}^{m}, x, q\right) \mid p^{m}(x), x\right) \geq \tau_{1}\right\}
\end{aligned}
$$

bound

$$
q \equiv Q_{Y_{1} \mid Y_{2} X}\left(\tau_{1} \mid y_{2}^{m}, x\right) \equiv \inf _{q}\left\{q: F_{Y_{1} \mid Y_{2} X}\left(q \mid y_{2}^{m}, x\right) \geq \tau_{1}\right\}
$$

respectively above and below, that is:

$$
q_{m} \leq q \leq q_{m-1}
$$

The final step is to obtain the relationships between the bounding functions, $q_{m-1}$ and $q_{m}$, and the structural function $h_{1}$.

First consider $q_{m}$ - a similar argument will apply for $q_{m-1}$. For any choice of $q^{*}$

$$
F_{\rho_{1} \mid \rho_{2} X}\left(h_{1}^{-1}\left(y_{2}^{m}, x, q^{*}\right) \mid p^{m}(x), x\right) \geq \tau_{1}
$$

if and only if

$$
Q_{\rho_{1} \mid \rho_{2} X}\left(\tau_{1} \mid p^{m}(x), x\right) \leq h_{1}^{-1}\left(y_{2}^{m}, x, q^{*}\right) .
$$

Therefore, recalling the definition of $h_{1}^{-1}$ given at the start of the Appendix,

$$
q_{m}=\inf _{q}\left\{q: Q_{\rho_{1} \mid \rho_{2} X}\left(\tau_{1} \mid p^{m}(x), x\right) \leq \sup _{p}\left\{p: h_{1}\left(y_{2}^{m}, x, p\right) \leq q\right\}\right\} .
$$


When $h_{1}$ is a strictly increasing function of $\rho_{1}$

$$
q_{m}=\inf _{q}\left\{q: Q_{\rho_{1} \mid \rho_{2} X}\left(\tau_{1} \mid p^{m}(x), x\right) \leq h_{1}^{-1}\left(y_{2}^{m}, x, q\right)\right\}
$$

and therefore

$$
q_{m}=h_{1}\left(y_{2}^{m}, x, Q_{\rho_{1} \mid \rho_{2} X}\left(\tau_{1} \mid p^{m}(x), x\right)\right) .
$$

It is now shown that the same expression obtains when $h_{1}$ is a weakly increasing function of $\rho_{1}$.

Consider

$$
q^{+} \equiv h_{1}\left(y_{2}^{m}, x, Q_{\rho_{1} \mid \rho_{2} X}\left(\tau_{1} \mid p^{m}(x), x\right)\right)
$$

which is a candidate value for $q_{m}$ in the weakly increasing case because,

$$
\sup _{p}\left\{p: h_{1}\left(y_{2}^{m}, x, p\right) \leq q^{+}\right\} \geq Q_{\rho_{1} \mid \rho_{2} X}\left(\tau_{1} \mid p^{m}(x), x\right),
$$

as Figure 4 makes clear.

In Figure 4 a non-decreasing caglad step function of $\rho_{1}, h_{1}$, is drawn with ends of steps at which $h_{1}$ is continuous from the left drawn as filled circles. The graph show a value of $Q_{\rho_{1} \mid \rho_{2} X}\left(\tau_{1} \mid p^{m}(x), x\right)$ and the candidate value $q^{+}$. The value of

$$
p^{+} \equiv \sup _{p}\left\{p: h_{1}\left(y_{2}^{m}, x, p\right) \leq q^{+}\right\}
$$

is indicated in Figure 4 and clearly $p^{+} \geq Q_{\rho_{1} \mid \rho_{2} X}\left(\tau_{1} \mid p^{m}(x), x\right)$, demonstrating that the inequality (L2.2) does hold.

It is evident from Figure 4 that for $q<q^{+}$there is no $p \geq Q_{\rho_{1} \mid \rho_{2} X}\left(\tau_{1} \mid p^{m}(x), x\right)$ such that

$$
\sup _{p}\left\{p: h_{1}\left(y_{2}^{m}, x, p\right) \leq q\right\} \geq Q_{\rho_{1} \mid \rho_{2} X}\left(\tau_{1} \mid p^{m}(x), x\right)
$$

and so $q_{m}=q^{+}$.

A similar argument produces the following expression for $q_{m-1}$.

$$
q_{m-1}=h_{1}\left(y_{2}^{m}, x, Q_{\rho_{1} \mid \rho_{2} X}\left(\tau_{1} \mid p^{m-1}(x), x\right)\right)
$$

Therefore, in the case in which $F_{\rho_{1} \mid \rho_{2} X}$ is non-decreasing in $\rho_{2}$ for $\rho_{2} \in\left(p^{m-1}(x), p^{m}(x)\right]$, there is the following inequality.

$$
\begin{gathered}
h_{1}\left(y_{2}^{m}, x, Q_{\rho_{1} \mid \rho_{2} X}\left(\tau_{1} \mid p^{m}(x), x\right)\right) \\
\leq Q_{Y_{1} \mid Y_{2} X}\left(\tau_{1} \mid y_{2}^{m}, x\right) \leq \\
h_{1}\left(y_{2}^{m}, x, Q_{\rho_{1} \mid \rho_{2} X}\left(\tau_{1} \mid p^{m-1}(x), x\right)\right)
\end{gathered}
$$

A similar argument for the case in which $F_{\rho_{1} \mid \rho_{2} X}$ is non-increasing in $\rho_{2}$ for $\rho_{2} \in$ $\left(p^{m-1}(x), p^{m}(x)\right]$ produces the reverse of this inequality

$$
h_{1}\left(y_{2}^{m}, x, Q_{\rho_{1} \mid \rho_{2} X}\left(\tau_{1} \mid p^{m-1}(x), x\right)\right)
$$




$$
\begin{gathered}
\leq Q_{Y_{1} \mid Y_{2} X}\left(\tau_{1} \mid y_{2}^{m}, x\right) \leq \\
h_{1}\left(y_{2}^{m}, x, Q_{\rho_{1} \mid \rho_{2} X}\left(\tau_{1} \mid p^{m}(x), x\right)\right)
\end{gathered}
$$

and combining (L2.3) and (L2.4) gives the result of the first part of the Lemma.

Suppose $Q_{\rho_{1} \mid \rho_{2} X}\left(\tau_{1} \mid r_{2}, x\right)$ is constant and equal to $r_{1}^{*}(x)$ for variation in $r_{2}$ over the interval $\left[p^{m-1}(x), p^{m}(x)\right]$. Then in each of (L2.3) and (L2.4) the lower and upper bounds are equal and so:

$$
\begin{aligned}
Q_{Y_{1} \mid Y_{2} X}\left(\tau_{1} \mid y_{2}^{m}, x\right) & =h_{1}\left(y_{2}^{m}, x, Q_{\rho_{1} \mid \rho_{2} X}\left(\tau_{1} \mid p^{m-1}(x), x\right)\right) \\
& =h_{1}\left(y_{2}^{m}, x, Q_{\rho_{1} \mid \rho_{2} X}\left(\tau_{1} \mid p^{m}(x), x\right)\right) \\
& =h_{1}\left(y_{2}^{m}, x, r_{1}^{*}(x)\right)
\end{aligned}
$$

which gives the result of the final part of the Lemma.

\section{Proof of Theorem 1.}

Consider the case in which for all $r_{1}, F_{\rho_{1} \rho_{2} X}\left(r_{1} \mid r_{2}, x\right)$ is a weakly increasing function of $r_{2}$ for $r_{2} \in R_{2}$. In this case, for all $\tau, Q_{\rho_{1} \rho_{2} X}\left(\tau \mid r_{2}, x\right)$ is a weakly decreasing function of $r_{2}$ for $r_{2} \in R_{2}$.

Lemma 2 implies the following inequalities.

$$
\begin{gathered}
Q_{Y_{1} \mid Y_{2} X}\left(\tau_{1} \mid y_{2}^{m}, x^{m-1}\right) \leq h_{1}\left(y_{2}^{m}, x^{m-1}, Q_{\rho_{1} \mid \rho_{2} X}\left(\tau_{1} \mid p^{m-1}\left(x^{m-1}\right), x^{m-1}\right)\right) \\
h_{1}\left(y_{2}^{m}, x^{m}, Q_{\rho_{1} \mid \rho_{2} X}\left(\tau_{1} \mid p^{m}\left(x^{m}\right), x^{m}\right)\right) \leq Q_{Y_{1} \mid Y_{2} X}\left(\tau_{1} \mid y_{2}^{m}, x^{m}\right)
\end{gathered}
$$

Because $p^{m}\left(x^{m}\right) \leq \tau_{2} \leq p^{m-1}\left(x^{m-1}\right)$ and in the case under consideration $Q_{\rho_{1} \rho_{2} X}\left(\tau_{1} \mid r_{2}, x\right)$ is a weakly decreasing function of $r_{2}$ for $r_{2} \in R_{2}$ there are the inequalities

$$
\begin{gathered}
Q_{\rho_{1} \rho_{2} X}\left(\tau_{1} \mid p^{m-1}\left(x^{m-1}\right), x^{m-1}\right) \leq Q_{\rho_{1} \rho_{2} X}\left(\tau_{1} \mid \tau_{2}, x^{m-1}\right) \\
Q_{\rho_{1} \rho_{2} X}\left(\tau_{1} \mid \tau_{2}, x^{m}\right) \leq Q_{\rho_{1} \rho_{2} X}\left(\tau_{1} \mid p^{m}\left(x^{m}\right), x^{m}\right)
\end{gathered}
$$

and because $h_{1}$ is monotonic in $\rho_{1}$ and normalised non-decreasing there are the following inequalities.

$$
\begin{gathered}
h_{1}\left(y_{2}^{m}, x^{m-1}, Q_{\rho_{1} \mid \rho_{2} X}\left(\tau_{1} \mid p^{m-1}\left(x^{m-1}\right), x^{m-1}\right)\right) \leq h_{1}\left(y_{2}^{m}, x^{m-1}, Q_{\rho_{1} \mid \rho_{2} X}\left(\tau_{1} \mid \tau_{2}, x^{m-1}\right)\right) \\
\quad h_{1}\left(y_{2}^{m}, x^{m}, Q_{\rho_{1} \mid \rho_{2} X}\left(\tau_{1} \mid \tau_{2}, x^{m}\right)\right) \leq h_{1}\left(y_{2}^{m}, x^{m}, Q_{\rho_{1} \mid \rho_{2} X}\left(\tau_{1} \mid p^{m}\left(x^{m}\right), x^{m}\right)\right)
\end{gathered}
$$

Combining (T1.1) with (T1.3) and (T1.2) with (T1.4), produces the following inequalities.

$$
\begin{gathered}
Q_{Y_{1} \mid Y_{2} X}\left(\tau_{1} \mid y_{2}^{m}, x^{m-1}\right) \leq h_{1}\left(y_{2}^{m}, x^{m-1}, Q_{\rho_{1} \mid \rho_{2} X}\left(\tau_{1} \mid \tau_{2}, x^{m-1}\right)\right) \\
h_{1}\left(y_{2}^{m}, x^{m}, Q_{\rho_{1} \mid \rho_{2} X}\left(\tau_{1} \mid \tau_{2}, x^{m}\right)\right) \leq Q_{Y_{1} \mid Y_{2} X}\left(\tau_{1} \mid y_{2}^{m}, x^{m}\right)
\end{gathered}
$$


Finally, under the covariation and exclusion restriction, B3 and B4 there is the following inequality for $x \in\left\{x^{m-1}, x^{m}\right\}$.

$$
Q_{Y_{1} \mid Y_{2} X}\left(\tau_{1} \mid y_{2}^{m}, x^{m-1}\right) \leq h_{1}\left(y_{2}^{m}, x, r_{1}^{*}\right) \leq Q_{Y_{1} \mid Y_{2} X}\left(\tau_{1} \mid y_{2}^{m}, x^{m}\right)
$$

Arguing as above for the case in which for all $r_{1}, F_{\rho_{1} \rho_{2} X}\left(r_{1} \mid r_{2}, x\right)$ is a weakly decreasing function of $r_{2}$ for $r_{2} \in R_{2}$ there is

$$
Q_{Y_{1} \mid Y_{2} X}\left(\tau_{1} \mid y_{2}^{m}, x^{m}\right) \leq h_{1}\left(y_{2}^{m}, x, r_{1}^{*}\right) \leq Q_{Y_{1} \mid Y_{2} X}\left(\tau_{1} \mid y_{2}^{m}, x^{m-1}\right)
$$

and combining the two cases gives the inequality of Theorem 1 .

\section{Proof of Theorem 2}

Since $\tau_{2}$ lies in $R_{2}$ and

$$
\begin{aligned}
\left(p^{m-1}\left(x^{m-1}\right), p^{m}\left(x^{m-1}\right)\right] & \subseteq R_{2} \\
\left(p^{m-1}\left(x^{m-1}\right), p^{m}\left(x^{m-1}\right)\right] & \subseteq R_{2}
\end{aligned}
$$

the constant value of $F_{\rho_{1} \rho_{2} X}\left(\tau_{1} \mid r_{2}, x\right)$ in Restriction B2* is $r_{1}^{*} \equiv F_{\rho_{1} \rho_{2} X}\left(\tau_{1} \mid \tau_{2}, x\right)$ which, by Restriction B3 is invariant with respect to choice of $x \in \tilde{x}_{m}$. The final result of Lemma 2 implies that

$$
\begin{aligned}
Q_{Y_{1} \mid Y_{2} X}\left(\tau_{1} \mid y_{2}^{m}, x^{m}\right) & =h_{1}\left(y_{2}^{m}, x, r_{1}^{*}\right) \\
Q_{Y_{1} \mid Y_{2} X}\left(\tau_{1} \mid y_{2}^{m}, x^{m-1}\right) & =h_{1}\left(y_{2}^{m}, x, r_{1}^{*}\right)
\end{aligned}
$$

the terms on the right hand sides of these equations being invariant with respect to choice of $x \in \tilde{x}_{m}$ by virtue of Restriction B4.

Lemma 3 is used in the proof of Theorem 3.

\section{Lemma 3}

Assume $h_{1}$ is strictly increasing in $\rho_{1}$. If, for all $r_{1}, F_{\rho_{1} \mid \rho_{2} X}\left(r_{1} \mid r_{2}, x\right)$ is weakly monotonic and non-constant in $r_{2}$ for $r_{2} \in\left(p^{m-1}(x), p^{m}(x)\right]$ over a set of values of nonzero measure then the inequalities of Lemma 2 are strong.

\section{Proof of Lemma 3}

Consider the case in which $F_{\rho_{1} \mid \rho_{2} X}\left(r_{1} \mid r_{2}, x\right)$ is non-decreasing for $r_{2} \in\left(p^{m-1}(x), p^{m}(x)\right]$. Then with $r_{1}=h_{1}^{-1}\left(y_{2}^{m}, x, y_{1}\right)$ :

$$
\begin{aligned}
& F_{\rho_{1} \mid \rho_{2} X}\left(r_{1} \mid r_{2}, x\right)=F_{\rho_{1} \mid \rho_{2} X}\left(r_{1} \mid p^{m-1}(x), x\right)+A^{m-1}\left(r_{1}, r_{2}, x\right) \\
& F_{\rho_{1} \mid \rho_{2} X}\left(r_{1} \mid r_{2}, x\right)=F_{\rho_{1} \mid \rho_{2} X}\left(r_{1} \mid p^{m}(x), x\right)+A^{m}\left(r_{1}, r_{2}, x\right)
\end{aligned}
$$

with $A^{m-1}>0$ and $A^{m}<0$ for some $r_{2} \in\left(p^{m-1}(x), p^{m}(x)\right]$ in a set of values of nonzero measure. The following strong inequalities result.

$$
F_{\rho_{1} \mid \rho_{2} X}\left(h_{1}^{-1}\left(y_{2}^{m}, x, y_{1}\right) \mid p^{m-1}(x), x\right)
$$




$$
\begin{gathered}
<F_{Y_{1} \mid Y_{2} X}\left(y_{1} \mid y_{2}^{m}, x\right)< \\
F_{\rho_{1} \mid \rho_{2} X}\left(h_{1}^{-1}\left(y_{2}^{m}, x, y_{1}\right) \mid p^{m}(x), x\right)
\end{gathered}
$$

The inequalities are reversed if $F_{\rho_{1} \mid \rho_{2} X}\left(r_{1} \mid r_{2}, x\right)$ is non-increasing for $r_{2} \in\left(p^{m-1}(x), p^{m}(x)\right]$.

Considering the solutions obtained on setting each term in the preceding inequalities equal to $\tau_{1}$ there is, arguing as in Lemma 2 ,

$$
\begin{gathered}
h_{1}\left(y_{2}^{m}, x, Q_{\rho_{1} \mid \rho_{2} X}\left(\tau_{1} \mid p^{m}(x), x\right)\right) \\
<Q_{Y_{1} \mid Y_{2} X}\left(\tau_{1} \mid y_{2}^{m}, x\right)< \\
h_{1}\left(y_{2}^{m}, x, Q_{\rho_{1} \mid \rho_{2} X}\left(\tau_{1} \mid p^{m-1}(x), x\right)\right)
\end{gathered}
$$

with the inequalities reversed if $F_{\rho_{1} \mid \rho_{2} X}\left(r_{1} \mid r_{2}, x\right)$ is non-increasing for $r_{2} \in\left(p^{m-1}(x), p^{m}(x)\right]$.

Thus the inequalities of Lemma 2 are strong under the stated conditions.

Remark A4. The conditions of Lemma 3 are somewhat stronger than required since the non-constancy of $F_{\rho_{1} \mid \rho_{2} X}\left(r_{1} \mid r_{2}, x\right)$ need only hold for $r_{1}$ in a sufficiently large region containing $\left.h_{1}^{-1}\left(y_{2}^{m}, x, Q_{Y_{1} \mid Y_{2} X}\left(\tau_{1} \mid y_{2}^{m}, x\right)\right)\right)$.

\section{Proof of Theorem 3}

Consider the case in which $F_{\rho_{1} \rho_{2} X}\left(r_{1} \mid r_{2}, x\right)$ is a weakly increasing function of $r_{2}$ for $r_{2} \in R_{2}$.

Under Restriction B2** the conditions of Lemma 3 are satisfied. Therefore in the proof of Theorem 1, the weak inequalities (T1.1) and (T1.2) are strong. Therefore the inequalities (T1.3) and (T1.4) are strong.

The same argument applies, with inequalities reversed, in the case in which $F_{\rho_{1} \rho_{2} X}\left(r_{1} \mid r_{2}, x\right)$ is a weakly decreasing function of $r_{2}$ for $r_{2} \in R_{2}$. It follows that the inequalities of Theorem 1 are strong and therefore that

$$
Q_{Y_{1} \mid Y_{2} X}\left(\tau_{1} \mid y_{2}^{m}, x^{m-1}\right) \neq Q_{Y_{1} \mid Y_{2} X}\left(\tau_{1} \mid y_{2}^{m}, x^{m}\right) .
$$


Figure 1: Estimated distribution functions of discrete years of schooling for quarter of birth 1 and 4

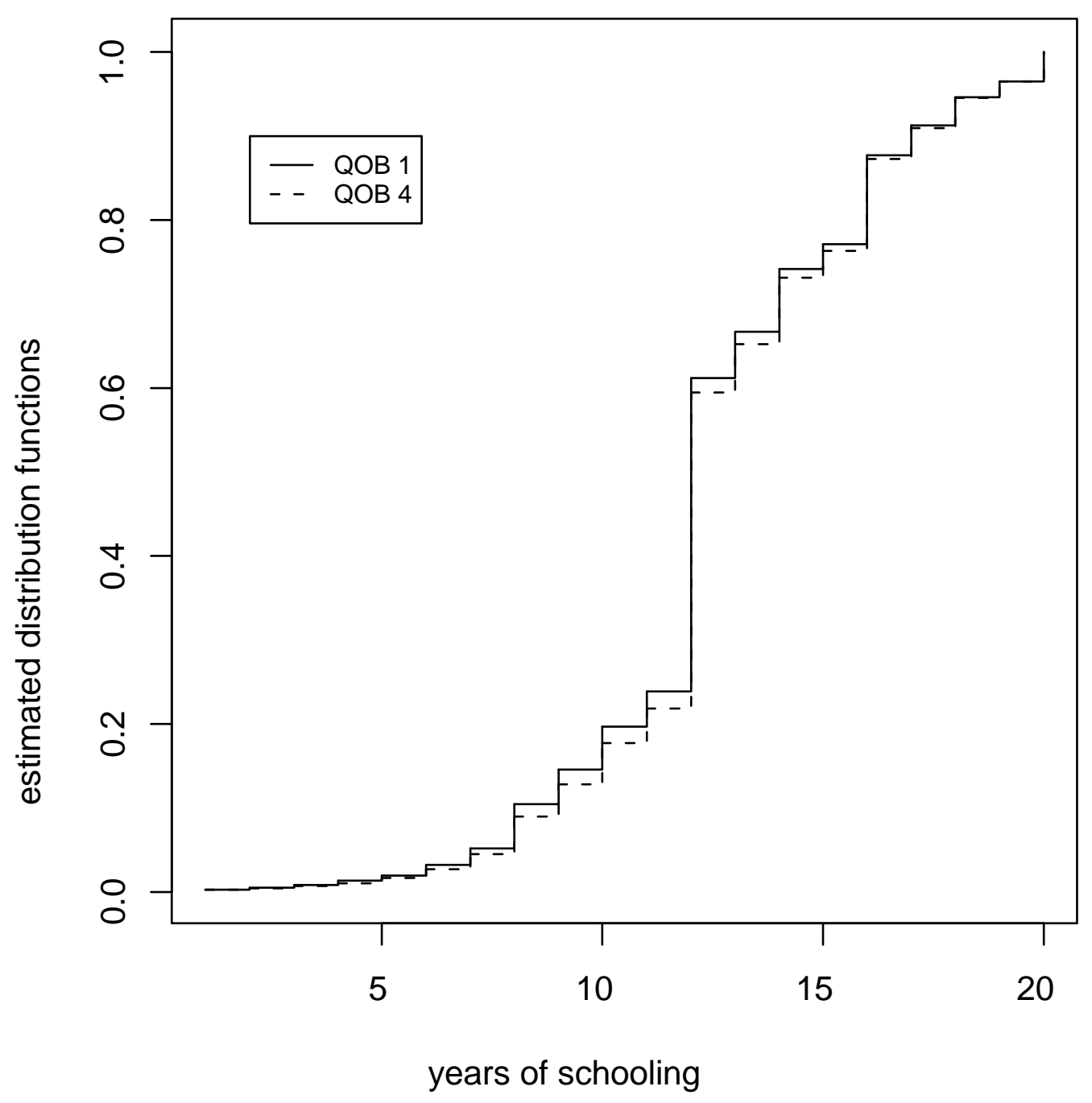


Figure 2: Conjectured distribution functions of continuous years of schooling for quarter of birth 1 and 4

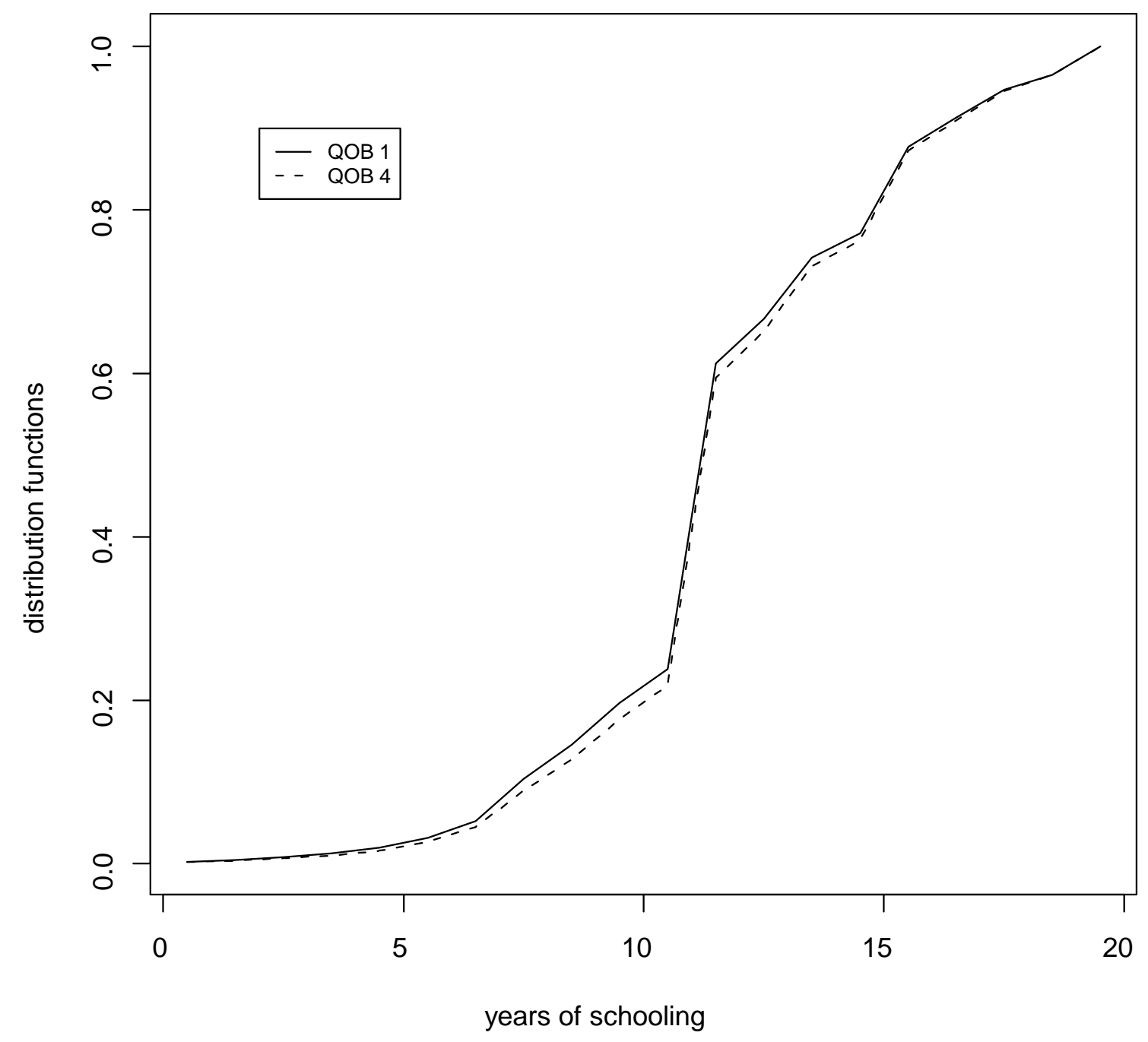


Figure 3: Conjectured distribution functions of continuous years of schooling ( 5 - 12 years) for quarter of birth 1 and 4

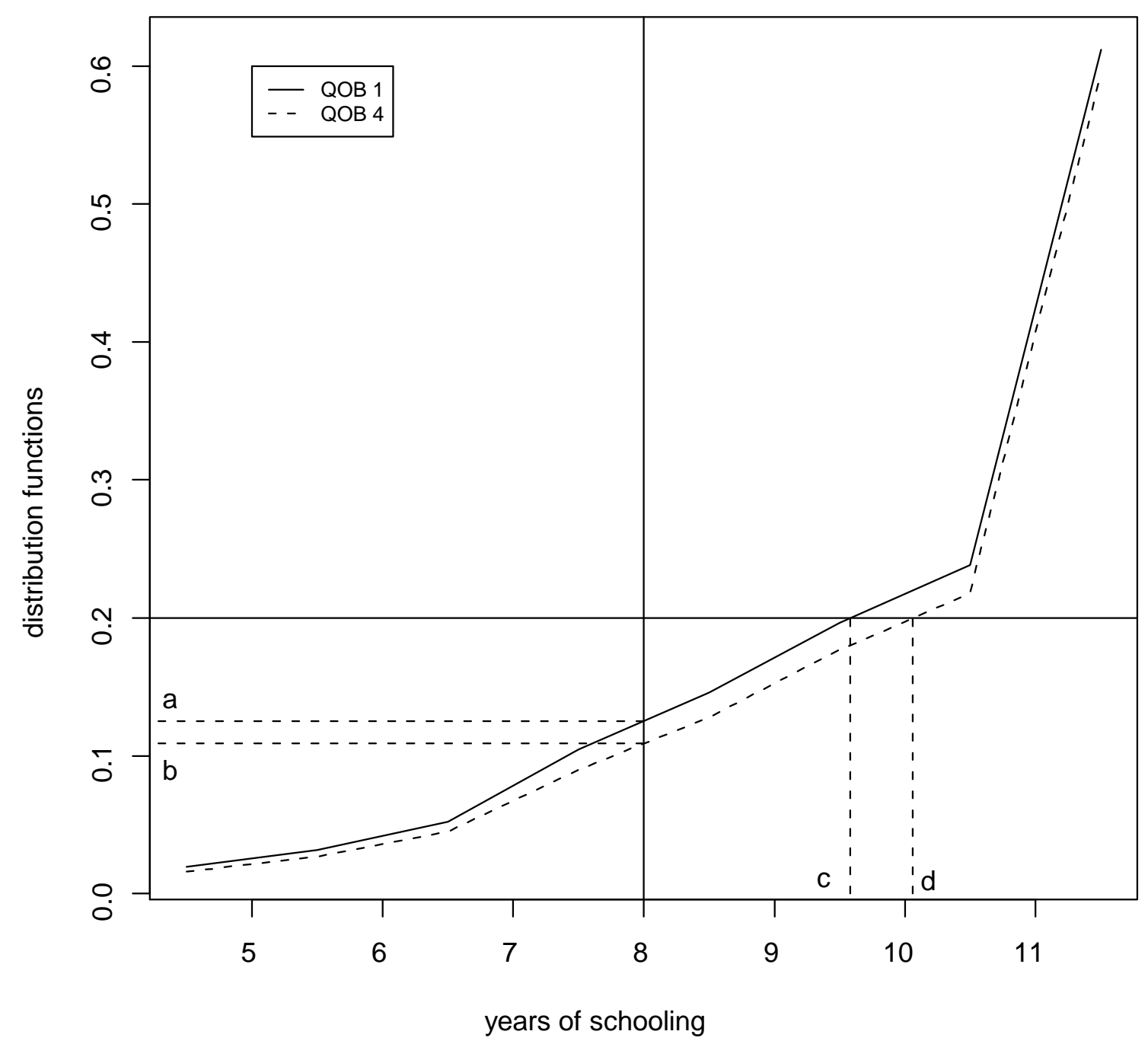




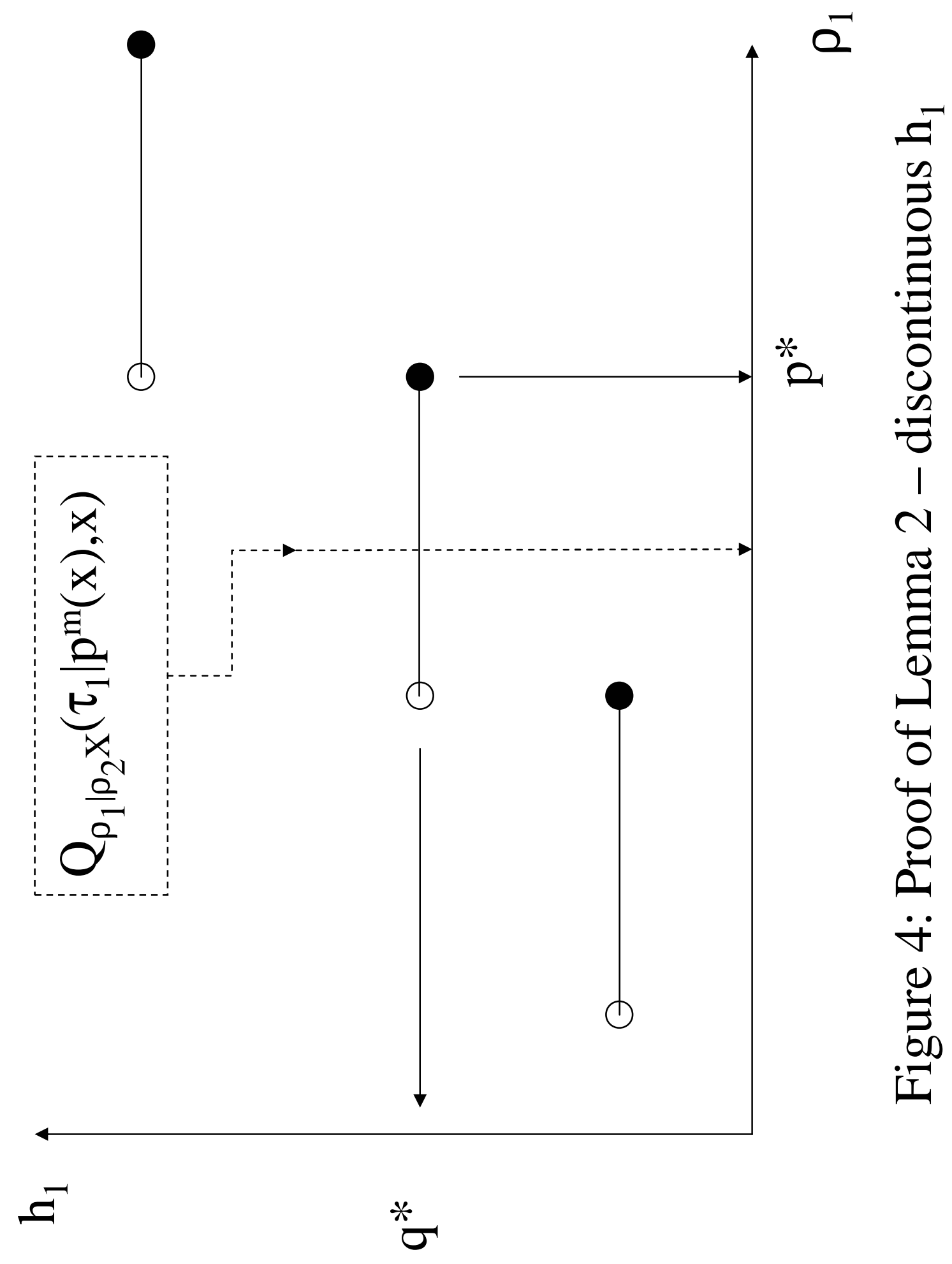

